\title{
On the Design and Multiplierless Realization of Perfect Reconstruction Triplet-Based FIR Filter Banks and Wavelet Bases
}

\author{
S. C. Chan, Member, IEEE, and K. S. Yeung
}

\begin{abstract}
This paper proposes new methods for the efficient design and realization of perfect reconstruction (PR) two-channel finite-impulse response (FIR) triplet filter banks (FBs) and wavelet bases. It extends the linear-phase FIR triplet FBs of Ansari et al. to include FIR triplet FBs with lower system delay and a prescribed order of $K$ regularity. The design problem using either the minimax error or least-squares criteria is formulated as a semidefinite programming problem, which is a very flexible framework to incorporate linear and convex quadratic constraints. The $K$ regularity conditions are also expressed as a set of linear equality constraints in the variables to be optimized and they are structurally imposed into the design problem by eliminating the redundant variables. The design method is applicable to linear-phase as well as low-delay triplet FBs. Design examples are given to demonstrate the effectiveness of the proposed method. Furthermore, it was found that the analysis and synthesis filters of the triplet FB have a more symmetric frequency responses. This property is exploited to construct a class of PR $M$-channel uniform FBs and wavelets with $M=2^{L}$, where $L$ is a positive integer, using a particular tree structure. The filter lengths of the two-channel FBs down the tree are approximately reduced by a factor of two at each level or stage, while the transition bandwidths are successively increased by the same factor. Because of the downsampling operations, the frequency responses of the final analysis filters closely resemble those in a uniform $\mathrm{FB}$ with identical transition bandwidth. This triplet-based uniform $M$-channel FB has very low design complexity and the PR condition and $K$ regularity conditions are structurally imposed. Furthermore, it has considerably lower arithmetic complexity and system delay than conventional tree structure using identical FB at all levels. The multiplierless realization of these FBs using sum-of-power-of-two (SOPOT) coefficients and multiplier block is also studied.
\end{abstract}

Index Terms-Design and multiplierless implementation, filter banks (FBs), low delay, perfect reconstruction (PR), semidefinite programming (SDP), triplet FBs, wavelets.

\section{INTRODUCTION}

$\mathbf{P}$ ERFECT reconstruction (PR) multirate filter banks (FBs) have important applications in signal analysis, signal coding, and the design of wavelet bases. One method for designing structural PR two-channel FBs and wavelets is to employ the triplet structure [8], [9], which can be viewed as three lifting steps [22]. The triplet FB can also be viewed as a

Manuscript received July 11, 2003; revised October 21, 2003. This paper was presented in part at the International Symposium on Circuits and Systems, Bangkok, Thailand, May 2003. This paper was recommended by Associate Editor E. A. B. da Silva.

The authors are with the Department of Electrical and Electronic Engineering, The University of Hong Kong, Hong Kong (e-mail: scchan@eee.hku.hk).

Digital Object Identifier 10.1109/TCSI.2004.832795 generalization of the two-channel structural PR FBs with two lifting steps [1]. In [9], Ansari et al. proposed two methods for designing structural PR two-channel linear-phase finite-impulse response (FIR) triplet FBs. One is based on the Lagrange halfband filters, where triplet FBs with maximal flatness can be obtained (or maximum $K$ regularity). The second method is based on Remez exchange algorithm, where an equiripple stopband response with user-defined cutoff frequencies can be obtained. The possibility of employing low-delay FIR FBs to reduce the system delay and method for imposing a prescribed $K$ regularity condition (which is equivalent to a certain number of zeros, respectively, at $\omega=\pi$ and $\omega=0$ of the low-pass $H_{0}(z)$ and high-pass $H_{1}(z)$ analysis filters) are not discussed. In fact, the latter involves a constrained minimax polynomial approximation problem, which cannot be handled directly by the Remez exchange algorithm. For linear-phase FIR FBs, it is possible to incorporate these linear equality constraints using linear programming. Unfortunately, linear programming is not applicable to general FIR FBs with approximate passband linear phase, which are useful in low-delay applications. Recently, Tay [8] proposes a Bernstein polynomial-based method for incorporating the $K$ regularity condition in linear-phase FIR triplet FBs. A least-squares (LS) approach was also proposed to determine the coefficients of the Bernstein polynomials through an iterative procedure, which optimizes a multiquadratic objective function.

In [3] and [29], we showed that the LS and minimax design of the structural PR two-channel FIR FBs in [1] with a prescribed $K$ regularity condition can be formulated respectively as a constrained LS and a semidefinite programming (SDP) problem. SDP has been successfully applied in areas such as control, logistic, digital filter design [5], digital signal processing, etc, and it can be solved efficiently in polynomial time using interior-point method [10]. It was shown in [3] that the SDP approach serves as a very flexible framework for designing the structural PR FBs and wavelets with LS and minimax design criteria, subject to linear and convex quadratic constraints. In general, the FBs and wavelets so obtained can be linear-phase or low-delay (approximate passband linear-phase) with a prescribed order of $K$ regularity. However, the magnitudes of the low-pass and high-pass analysis filters of this structure at $\omega=$ $\pi / 2$ are restricted to be 0.5 and 1 , respectively. In other words, the magnitude characteristics of the low- and high-pass analysis filters are not so symmetric with respect to each other. On the other hand, in the triplet structure, these magnitudes can be controlled by a set of parameters. In particular, they can be 
made equal to each other so that a more symmetric frequency responses of the analysis filter pair are obtained.

In this paper, we propose a new method to design the PR FIR triplet FBs and wavelets with a prescribed order of $K$ regularity. First of all, it is shown that the constrained design problem with a weighted minimax error or LS criteria can be formulated as an SDP problem. This can be viewed as an extension of the SDP approach that we have reported previously for designing two-channel structural PR FIR FBs [3], [29]. The proposed approach is rather general and it can be applied to the linear-phase and the low-delay cases. Design results showed that the SDP approach offers a comparable performance as [9] for the linear-phase case. Furthermore, it is possible to design low-delay triplet FBs and wavelets with a prescribed order of $K$ regularity. Due to the improved frequency characteristics (more symmetric frequency responses) of the analysis and synthesis filters of the triplet FBs, they are more amendable to be cascaded in a tree structure to form an $M$-channel uniform FB or wavelet, where $M=2^{L}$, and $L$ is the number of tree levels. It will be shown later that by properly choosing the filter lengths and transition bandwidths of the triplet FBs in the tree structure, uniform FBs and wavelets with very good frequency characteristics can be generated. On the other hand, if the structural PR FB in [1] is used, then the frequency characteristics will be considerably degraded after cascading, due to the magnitude constraints at $\omega=\pi / 2$ mentioned earlier. Besides, the multiplierless realization of the triplet-based FBs using the sum-of-powers-of-two (SOPOT) coefficients [23] and the multipler block (MB) [27] is studied.

One motivation of cascading triplet FBs to form a uniform $\mathrm{FB}$ is that the PR and regularity properties will be preserved. In fact, the $M$-channel ( $M=2^{L}$ ) FB is PR if all the two-channel FBs in the tree are PR [13]. Moreover, the $M$-channel FB is a wavelet FB if all the two-channel FBs in the tree are wavelet FBs. However, if identical two-channel FBs are cascaded together, then the system delay will become very large and the resulting frequency characteristics do not usually resemble an ideal uniform $M$-channel FB. Recently, Xie et al. [15] extends an observation of Fliege [17] and Nayebi et al. [18] and study a simple method for designing tree-structured linear-phase and low-delay $M$-channel FBs with $M=2^{L}$. More precisely, the filter lengths of the two-channel FBs down the tree are approximately reduced by a factor of two at each level or stage, while the cutoff frequencies are successively increased by the same factor. Because of the downsampling operations, the final analysis filters resemble closely those in a uniform FB with identical transition bandwidth (see, for example, Figs. 5(c) and 7 shown later). In [15], the PR two-channel FBs are derived from the lattice structure proposed in [19]. They are obtained independently by nonlinear constrained optimization to meet the given frequency characteristics and transition bandwidth requirements. This structure can be used to construct PR $M$-channel wavelets if all the two-channel PR FBs are wavelets, say by imposing the PR and $K$ regularity conditions. Another advantage of this structure is that its complexity and system delay are much lower than a conventional tree structure when identical two-channel FBs are used at all levels of the tree, especially when the depth of the tree increases. By employing the triplet FBs as the com-

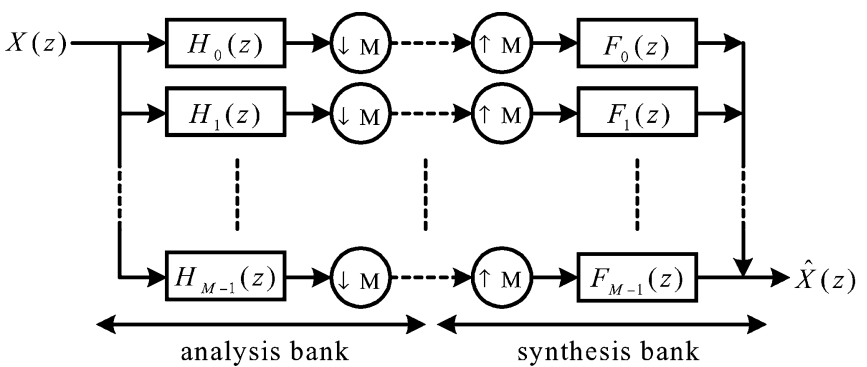

(a)

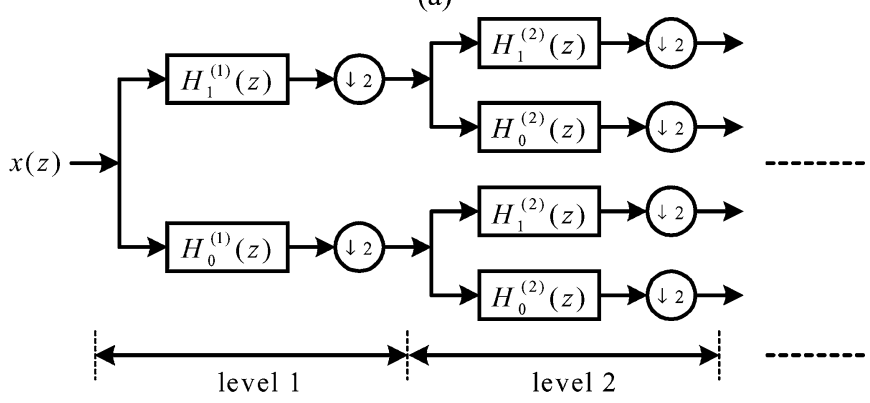

(b)

Fig. 1. (a) Critically decimated $M$-channel uniform FB. (b) Two-level maximally decimated tree-structured analysis FB.

ponent two-channel FBs in this particular tree structure, the PR condition can be structurally imposed. Further, the component FBs can be designed independently using SDP to yield more symmetric frequency responses. After cascading, linear-phase as well as low-delay $M$-channel FBs and wavelets with very good frequency characteristics are achieved. The design and implementation complexities of this type of $M$-channel uniform FB are rather low, thanks to the triplet structure. It is also interesting to note that the linear-phase scaling functions and wavelets generated by this tree structure are all symmetric while those obtained in [12] are either symmetric or asymmetric.

The rest of this paper is organized as follows. Section II is devoted to the design of PR two-channel FIR triplet FBs and wavelets. The problem formulation and several design examples will be given. In Section III, the construction of the PR tree-structured $M$-channel uniform FBs and wavelets using the triplet FBs is described. Several design examples are also given. This is followed by the multiplierless realization of the triplet FB in Section IV. Finally, conclusion is drawn in Section V.

\section{PR TWO-ChANNEL FIR TRIPLET FBs AND WAVELET BASES}

The general structure of an $M$-channel uniform FB is shown in Fig. 1(a). The $z$ transform of the output of a two-channel FB, i.e., $M=2$, can be expressed as

$$
\begin{aligned}
\hat{X}(z)= & {\left[H_{0}(z) F_{0}(z)+H_{1}(z) F_{1}(z)\right] X(z) } \\
& +\left[H_{0}(-z) F_{0}(z)+H_{1}(-z) F_{1}(z)\right] X(-z) .
\end{aligned}
$$

The two-channel FB is PR if

$$
\begin{aligned}
H_{0}(z) F_{0}(z)+H_{1}(z) F_{1}(z) & =z^{-n_{d}} \\
H_{0}(-z) F_{0}(z)+H_{1}(-z) F_{1}(z) & =0
\end{aligned}
$$




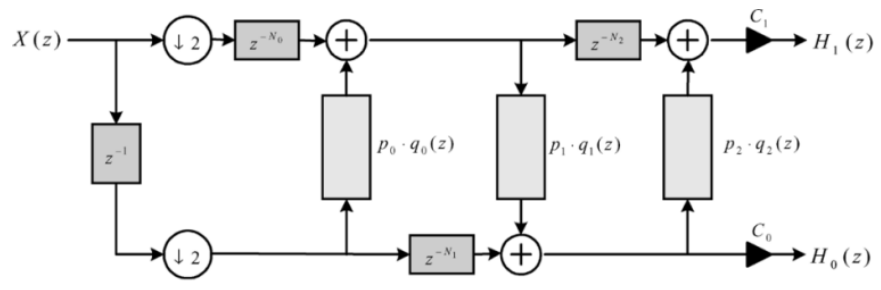

(a)

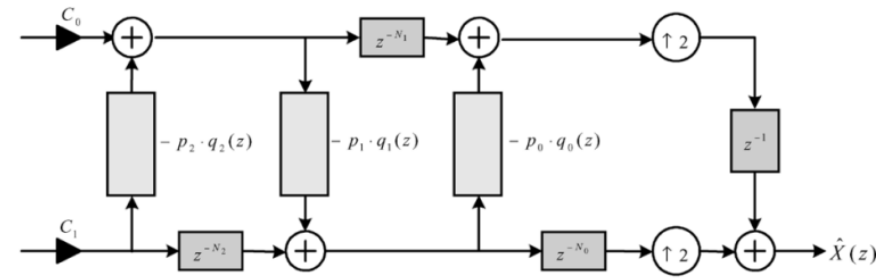

(b)

Fig. 2. Structure of the two-channel triplet-based FBs. (a) Analysis bank. (b) Synthesis bank.

where $n_{d}$ is an integer. Substituting (2.2) into (2.1), it can be seen the input and output of the FB are identical up to a time delay of $n_{d}$ samples, i.e., $\hat{x}(n)=x\left(n-n_{d}\right)$.

\section{A. Problem Formulation}

Fig. 2 shows the structure of the analysis and synthesis filters of the triplet FB. It is parameterized by three subfilters $q_{i}(z)$, three delay parameters $N_{i}$, and five constant parameters $p_{i}$ and $C_{m}$ for $i=0,1,2$ and $m=0,1$. The triplet FB is structural PR for arbitrary choice of these subfilters, which can be chosen as linear-phase/low-delay FIR or IIR filters. It is a generalization of the structural PR FB of Phoong et al. [1], which consists only of $q_{0}(z)$ and $q_{1}(z)$. Here, we shall consider the design of the subfilters $q_{i}(z)$ for linear-phase as well as low-delay FIR triplet FB and a prescribed order of $K$ regularity using SDP. In particular, it will be shown that PR FIR triplet FBs with equiripple or LS stopband response and a prescribed order of $K$ regularity can be formulated as a SDP problem. This approach is rather general that it is applicable to both the linear-phase and low-delay cases.

Although there are other possible choices of the parameters $p_{i}$ and $C_{m}$ in the triplet $\mathrm{FB}$, a very good choice is [8]: $p_{0}=-p$, $p_{1}=1 /(1+p), p_{2}=\left(p^{2}-1\right) / 2, C_{0}=(1+p) / 2$ and $C_{1}=1 /(1+p)$. With this particular choice, the magnitudes of the low-pass and high-pass analysis/synthesis filters at $\omega=$ $\pi / 2$ can be parameterized by a single parameter $p$. Further, by choosing $p$ as $\sqrt{2}-1$, the magnitudes of the analysis filter pair at $\omega=\pi / 2$ will be equal to $1 / \sqrt{2}$ so that more symmetric frequency response of the analysis filters can be obtained. To proceed further, let us consider the $z$ transform of the analysis filter pair as follows:

$$
\begin{aligned}
H_{0}(z)= & \frac{1}{2}(1+p) z^{-\left(2 N_{1}+1\right)} \\
& +\frac{1}{2} q_{1}\left(z^{2}\right)\left[z^{-2 N_{0}}-z^{-1} p \cdot q_{0}\left(z^{2}\right)\right] \\
H_{1}(z)= & \frac{z^{-2 N_{0}}-z^{-1} p \cdot q_{0}\left(z^{2}\right)}{1+p} z^{-2 N_{2}} \\
& -\frac{1-p}{1+p} q_{2}\left(z^{2}\right) H_{0}(z)
\end{aligned}
$$

Similarly, the $z$ transform of the synthesis filter pair can be written as

$$
\begin{aligned}
F_{0}(z)= & H_{1}(-z) \\
= & \frac{z^{-2\left(N_{0}+N_{2}\right)}+z^{-\left(2 N_{2}+1\right)} p q_{0}\left(z^{2}\right)}{1+p} \\
& -\frac{1-p}{1+p} q_{2}\left(z^{2}\right) H_{0}(-z) \\
F_{1}(z)= & -H_{0}(-z) \\
= & -q_{1}\left(z^{2}\right) z^{-2 N_{0}}+H_{0}(z) .
\end{aligned}
$$

Using (2.3) and (2.2), one can verify that the system delay of the FB is given by $n_{d}=2 N_{0}+2 N_{1}+2 N_{2}+1$. Let $\omega_{p i}, i=0,1,2$, be the passband cutoff frequency of $q_{i}(z)$. To design the low-pass analysis filter $H_{0}(z)$, we first design the subfilter $q_{0}(z)$ with the following desired frequency response:

$$
q_{0}^{(d)}\left(e^{j 2 \omega}\right)=e^{-j\left(2 N_{0}-1\right) \omega}, \quad \text { for } \omega \in\left[0, \omega_{p 0}\right] .
$$

Once $q_{0}(z)$ is designed, one can design $q_{1}(z)$ with the following desired frequency response:

$$
\begin{aligned}
& q_{1}^{(d)}\left(e^{j 2 \omega}\right)=\frac{(1+p) e^{-j\left(2 N_{1}+1\right) \omega}}{e^{-j \omega} p \cdot q_{0}\left(e^{j 2 \omega}\right)-e^{-j 2 N_{0} \omega}}, \\
& \text { for } \omega \in\left[\pi-\omega_{p 1}, \pi\right] .
\end{aligned}
$$

From (2.4a), we can see that $q_{0}\left(e^{j 2 \omega}\right)$ approximates $e^{-j\left(2 N_{0}-1\right) \omega}$ for $0<\omega<\pi / 2$. Due to the periodic nature of $q_{0}\left(e^{j 2 \omega}\right)$, for $\pi / 2<\omega<\pi, q_{0}\left(e^{j 2 \omega}\right)$ becomes anti-phase with $e^{-j 2 N_{0} \omega}$. Therefore, the term inside the square bracket in $(2.3 \mathrm{a})$ approximates $(1-p) e^{-j 2 N_{0} \omega}$ in the passband, $0<\omega<\pi / 2$, and becomes $(1+p) e^{-j 2 N_{0} \omega}$ in the stopband, $\pi / 2<\omega<\pi$. As for $q_{1}\left(e^{j 2 \omega}\right)$ in (2.4b), the product $(1 / 2) q_{1}\left(e^{j 2 \omega}\right)\left[e^{-j 2 N_{0} \omega}-e^{-j \omega} p \cdot q_{0}\left(e^{j 2 \omega}\right)\right]$ in (2.3a) will approximate $(1 / 2)(1-p) e^{-j\left(2 N_{1}+1\right) \omega}$ in $0<\omega<\pi / 2$. Therefore, $H_{0}\left(e^{j \omega}\right)$ is approximately equal to $e^{-j\left(2 N_{1}+1\right) \omega}$ in the passband. In the stopband, $(1 / 2) q_{1}\left(e^{j 2 \omega}\right)\left[e^{-j 2 N_{0} \omega}-e^{-j \omega} p \cdot q_{0}\left(e^{j 2 \omega}\right)\right]$ in (2.3a) will approximate $-(1 / 2)(1+p) e^{-j\left(2 N_{1}+1\right) \omega}$, which cancels with the signal delay to give a value nearly equal to zero.

Given $H_{0}(z)$ and $q_{0}(z)$, the high-pass analysis filter $H_{1}(z)$ can be obtained by designing $q_{2}(z)$ with the following desired frequency response:

$$
\begin{array}{r}
q_{2}^{(d)}\left(e^{j 2 \omega}\right)=\frac{e^{-j\left(2 N_{0}+2 N_{2}\right) \omega}-e^{-j\left(2 N_{2}+1\right) \omega} p \cdot q_{0}\left(e^{j 2 \omega}\right)}{(1-p) H_{0}\left(e^{j \omega}\right)} \\
\text { for } \omega \in\left[0, \omega_{p 2}\right] .
\end{array}
$$

Similarly, it can be verified that $H_{1}\left(e^{j \omega}\right)$ will approximate zero and $e^{-j\left(2 N_{1}+2 N_{2}\right) \omega}$, respectively, in $0<\omega<\pi / 2$ and $\pi / 2<$ $\omega<\pi$, for the given choice of $q_{i}(z)$. These conditions for the low-delay triplet FB will reduce to the linear-phase triplet FBs considered in [9], if $q_{i}(z)$ 's are linear-phase. In this case, $L_{q 0}=$ $2 N_{0}, L_{q 1}=2\left(N_{1}-N_{0}+1\right)$, and $L_{q 2}=2\left(N_{0}-N_{1}+N_{2}\right)$, where $L_{q i}, i=0,1,2$, are, respectively, the subfilter length of $q_{i}(z)$. Like the low-delay structural PR two-channel FB in [2], the subfilter lengths of the proposed low-delay triplet FB are not constrained by the delay parameters $N_{0}, N_{1}$, and $N_{2}$, and they 
can be increased independently to provide a higher stopband attenuation. Next, we shall show that the $K$ regularity conditions can be written as a set of linear equality constraints in the subfilter coefficients of $q_{i}(z)$, and the design problem can be solved using SDP.

\section{B. K-Regularity Condition}

To construct wavelet bases from triplet FBs, the analysis filter pair $H_{m}(z), m=0,1$, should possess at least one zero at $\omega=\pi$ and $\omega=0$, respectively. Let $K_{0}$ and $K_{1}$ be the number of zeros to be imposed, respectively, at $\omega=\pi$ and $\omega=0$ for $H_{0}(z)$ and $H_{1}(z)$ with $K_{0} \geq K_{1} \geq 1$. This is equivalent to

$$
\left.\frac{d^{k_{0}}}{d \omega^{k_{0}}} H_{0}\left(e^{j \omega}\right)\right|_{\omega=\pi}=\left.\frac{d^{k_{1}}}{d \omega^{k_{1}}} H_{1}\left(e^{j \omega}\right)\right|_{\omega=0}=0
$$

for $k_{0}=0, \ldots, K_{0}-1$ and $k_{1}=0, \ldots, K_{1}-1$. In general, the number of zeros imposed for the analysis filters is closely related to the following halfband filters $H_{q i}(z), i=0,1,2$ :

$$
H_{q i}(z)=\frac{\left[z^{-1} q_{i}\left(z^{2}\right)+z^{-2 N_{q i}}\right]}{2}
$$

where $N_{q 0}=N_{0}, N_{q 1}=N_{1}-N_{0}+1, N_{q 2}=N_{2}+N_{0}-N_{1}$. Substituting (2.6) into (2.3a), one gets

$$
H_{0}(z)=-p \cdot q_{1}\left(z^{2}\right) H_{q 0}(z)+(1+p) z^{-2 N_{0}+1} H_{q 1}(z) .
$$

It can be seen that if $H_{q 0}(z)$ and $H_{q 1}(z)$ have respectively $K_{q 0}$ and $K_{q 1}$ zeros at $z=-1$, then $H_{0}(z)$ has at least $K_{0}$ zeros at $z=-1$ with $K_{0}=\min \left\{K_{q 0}, K_{q 1}\right\}$. Similarly, $F_{0}(z)$ can be written as

$$
\begin{aligned}
& F_{0}(z) \\
& =\frac{2 p \cdot z^{-2 N_{2}} H_{q 0}(z)+(1-p) q_{2}\left(z^{2}\right) H_{0}(z)}{1+p} \\
& \quad-\frac{4(1-p) z^{-2\left(N_{0}-1\right)} H_{q 1}(z) H_{q 2}(z)}{1+p} \\
& \quad+\frac{2(1-p)\left[z^{-2 N_{1}} H_{q 2}(z)+z^{-2\left(2 N_{0}+N_{2}-N_{1}-1\right)} H_{q 1}(z)\right]}{1+p} .
\end{aligned}
$$

Again, it can be seen that if $H_{q i}(z), i=0,1,2$, have respectively $K_{q i}$ zeros at $z=-1$ respectively, then $F_{0}(z)$ has at least $K_{1}$ zeros at $z=-1$ with $K_{1}=\min \left\{K_{q 0}, K_{q 1}, K_{q 2}\right\}$. In this work, $q_{i}(z)$ 's are assumed to be a general FIR filter with $q_{i}(z)=\sum_{n=0}^{L_{q i}-1} q_{i, n} z^{-n}$. The $K$ regularity conditions can be obtained by considering the following form of the halfband filter:

$$
H_{q i}(z)=\frac{\left[z^{-2 L_{q i}+1} \widetilde{H}_{q i}(z)\right]}{2} \text { with } L_{q i}>N_{q i}
$$

where $\widetilde{H}_{q i}(z)=\sum_{n=0}^{L_{q i}-1} q_{i, n} z^{2\left(L_{q i}-n-1\right)}+z^{2\left(L_{q i}-N_{q i}\right)-1}$. The conditions for $H_{q i}(z)$ to have $K_{q i}$ zeros at $z=-1$ are equivalent to

$$
\left.\frac{d^{k_{q i}}}{d z^{k_{q i}}} \widetilde{H}_{q i}(z)\right|_{z=-1}=0
$$

Expanding (2.10) and after slight manipulation, one gets a set of linear equality constraints as follows:

$$
\begin{array}{r}
\sum_{n-0}^{L_{q i}-1-\left\lceil k_{q i} / 2\right\rceil} q_{i, n} \frac{\left(2\left(L_{q i}-n\right)-2\right) !}{\left(2\left(L_{q i}-n\right)-2-k_{q i}\right) !} \\
=\frac{\left(2\left(L_{q i}-N_{q i}\right)-1\right) !}{\left(2\left(L_{q i}-N_{q i}\right)-1-k_{q i}\right) !}
\end{array}
$$

for $k_{q i}=0,1, \ldots, K_{q i}-1$, where the operator $\lceil\alpha\rceil$ denotes the nearest integer greater than or equal to $\alpha$. Equation (2.11a) can also be written in the following matrix form:

$$
A_{i} \cdot \boldsymbol{q}_{i}=B_{i}
$$

where

$$
\begin{aligned}
{\left[\boldsymbol{A}_{i}\right]_{k_{q i}, n} } & =\left(2\left(L_{q i}-n\right)-2\right) ! /\left(2\left(L_{q i}-n\right)-2-k_{q i}\right) ! \\
{\left[\boldsymbol{q}_{i}\right]_{n} } & =q_{i, n} \\
{\left[\boldsymbol{B}_{i}\right]_{k_{q i}} } & =\left(2\left(L_{q i}-N_{q i}\right)-2\right) ! /\left(2\left(L_{q i}-N_{q i}\right)-2-k_{q i}\right) !
\end{aligned}
$$

Here, $[\boldsymbol{A}]_{n, m}$ denotes the $(n, m)$ th entry of matrix $\boldsymbol{A}$. This will be used to eliminate the redundant variables in the SDP design method to be described in the following subsection.

\section{SDP Design}

In this section, we shall show that the design of the subfilters $q_{i}\left(e^{j \omega}\right)$ with minimax and LS error criteria and a prescribed order of $K$ regularity can be formulated as a SDP problem.

1) Weighted Minimax Error Criterion: To minimize the maximum ripple of the approximation error is equivalent to the following:

$$
\min _{\boldsymbol{q}_{i}} \max W(\omega)\left|\boldsymbol{q}_{i}^{T} \cdot \boldsymbol{e}_{i}-q_{i}^{(d)}\left(e^{j \omega}\right)\right|, \text { for } \omega \in\left[0,2 \omega_{p i}\right]
$$

where $\boldsymbol{q}_{i}=\left[q_{i, 0} q_{i, 1} \cdots q_{i, L_{q i}-1}\right]^{T}$ is the impulse response of $q_{i}(z) ; \boldsymbol{e}_{i}=\left[1 e^{-j \omega} \cdots e^{-j\left(L_{q i}-1\right) \omega}\right]^{T} ; q_{i}^{(d)}\left(e^{j \omega}\right)$ is the desired frequency response defined in (2.4); $W(\omega)$ is a positive weighting function. To solve (2.12) using SDP, we densely discretize $\omega$ over the band of interest $\omega \in\left[0,2 \omega_{p i}\right]$ into a set of frequency points $\omega_{k}, k=1, \ldots, K$. It yields

$$
\min _{\boldsymbol{q}_{i}} \delta_{i} \text { subject to } \alpha_{R, i}^{2}\left(\omega_{k}\right)+\alpha_{I, i}^{2}\left(\omega_{k}\right) \leq \delta_{i}
$$

where

$$
\begin{aligned}
\alpha_{R, i}\left(\omega_{k}\right) & =W(\omega)\left|\boldsymbol{q}_{\boldsymbol{i}}^{T} \cdot \boldsymbol{c}_{i}-\operatorname{Re}\left\{q_{i}^{(d)}\left(e^{j \omega_{k}}\right)\right\}\right| \\
\boldsymbol{c}_{i} & =\left[1 \cos \left(\omega_{k}\right) \cdots \cos \left(\left(L_{q i}-1\right) \omega_{k}\right)\right]^{T} \\
\alpha_{I, i}\left(\omega_{k}\right) & =W(\omega)\left|\boldsymbol{q}_{i}^{T} \cdot \boldsymbol{s}_{i}+\operatorname{Im}\left\{q_{i}^{(d)}\left(e^{j \omega_{k}}\right)\right\}\right| \\
\boldsymbol{s}_{i} & =\left[0 \sin \left(\omega_{k}\right) \cdots \sin \left(\left(L_{q i}-1\right) \omega_{k}\right)\right]^{T} .
\end{aligned}
$$

Using the Schur complement [11], it can be shown that (2.13a) is equivalent to

$$
\min _{\boldsymbol{q}_{i}} \delta_{i} \text { subject to } \boldsymbol{\Gamma}_{k}\left(\boldsymbol{q}_{i}\right) \geq 0
$$


where

$$
\boldsymbol{\Gamma}_{k}\left(\boldsymbol{q}_{i}\right)=\left(\begin{array}{ccc}
\delta_{i} & \alpha_{R, i}\left(\omega_{k}\right) & \alpha_{I, i}\left(\omega_{k}\right) \\
\alpha_{R, i}\left(\omega_{k}\right) & 1 & 0 \\
\alpha_{I, i}\left(\omega_{k}\right) & 0 & 1
\end{array}\right)
$$

and $C \geq 0$ means that matrix $\boldsymbol{C}$ is positive semidefinite. Since $\boldsymbol{\Gamma}_{k}\left(\boldsymbol{q}_{i}\right)$ is affine in $\boldsymbol{q}_{i}$, it is equivalent to a set of linear-matrix inequalities (LMI) [11]. In order to simultaneously solve the SDP problem in (2.13b) and the $K$ regularity conditions mentioned in Section II-B, the dependent variables defined by the linear equality constraints $(2.11 \mathrm{~b})$ can be expressed as a linear combination of independent variables. The number of variables to be optimized is therefore reduced. It not only speeds up the optimization process but also structurally imposes the $K$ regularity conditions. To remove the redundant variables, let $r_{i}$ be the number of redundant variables in $q_{i}(z)$. Equation (2.11b) can be rewritten as

$$
\left[\begin{array}{ll}
\boldsymbol{A}_{i}, L_{q i}-r_{i} & \boldsymbol{A}_{i, r_{i}}
\end{array}\right] \cdot\left[\begin{array}{c}
\boldsymbol{q}_{i}, L_{q i}-r_{i} \\
\boldsymbol{q}_{i, r_{i}}
\end{array}\right]=\boldsymbol{B}_{i}
$$

where

$$
\begin{aligned}
\boldsymbol{A}_{i} & =\left[\begin{array}{ll}
\boldsymbol{A}_{i}, L_{q i}-r_{i} & \boldsymbol{A}_{i, r_{i}}
\end{array}\right] \\
\boldsymbol{q}_{i} & =\left[\begin{array}{c}
\boldsymbol{q}_{i}, L_{q i}-r_{i} \\
\boldsymbol{q}_{i, r_{i}}
\end{array}\right] .
\end{aligned}
$$

Using (2.14), $\boldsymbol{q}_{i}$ can be rewritten in terms of $\boldsymbol{q}_{i}, L_{q i}-r_{i}$ as

$$
\boldsymbol{q}_{i}=\left[\begin{array}{c}
O_{i}, L_{q i}-r_{i} \\
\boldsymbol{A}_{i, r_{i}}^{-1} \boldsymbol{B}_{\boldsymbol{i}}
\end{array}\right]+\left[\begin{array}{c}
\boldsymbol{I}_{i}, L_{q i}-r_{i} \\
-\boldsymbol{A}_{i, r_{i}}^{-1} \boldsymbol{A}_{i}, L_{q i}-r_{i}
\end{array}\right] \boldsymbol{q}_{i}, L_{q i}-r_{i}
$$

where $\boldsymbol{O}_{N}$ is an $(N \times 1)$ null vector and $\boldsymbol{I}_{N}$ is an $(N \times N)$ identity matrix. Substituting (2.15) into (2.13b) and defining the augmented variable $\boldsymbol{x}_{i}^{T}=\left[\begin{array}{ll}\delta_{i} & \boldsymbol{q}_{i}, L_{q i}-r_{i}\end{array}\right]$, the optimization problem in $(2.13 \mathrm{~b})$ can be cast into the following standard SDP problem:

$$
\min _{\boldsymbol{x}_{i}} \boldsymbol{u}_{i}^{T} \cdot \boldsymbol{x}_{i} \text { subject to } \boldsymbol{F}\left(\boldsymbol{x}_{\boldsymbol{i}}\right) \geq 0
$$

where $\boldsymbol{u}_{\boldsymbol{i}}^{T}=\left[\begin{array}{ll}1 & \boldsymbol{O}_{L_{q i}-r_{i}}^{T}\end{array}\right] ; \boldsymbol{F}\left(\boldsymbol{x}_{i}\right)=\operatorname{diag}\left(\boldsymbol{\Gamma}_{1}\left(\boldsymbol{x}_{i}\right), \ldots, \boldsymbol{\Gamma}_{K}\left(\boldsymbol{x}_{i}\right)\right)$. Theoretically, it is possible to determine whether a feasible solution exists for the SDP problem (2.16), and if so, it is possible to determine the global optimal solution, since the problem is convex. Moreover, the SDP problem is very general in that other design criteria such as LS, and LS with peak constraints can be employed, possibly with linear and convex quadratic constraints.

2) Weighted LS Error Criterion With Peak Error Constraints: Another common criterion to be minimized is the following LS objective function:

$$
\begin{aligned}
E_{L S}\left(\boldsymbol{q}_{i}\right) & =\int_{\Omega} W(\omega) \cdot\left|q_{i}\left(e^{j \omega}\right)-q_{i}^{(d)}\left(e^{j \omega}\right)\right|^{2} d \omega \\
& =\boldsymbol{q}_{i}^{\boldsymbol{T}} \boldsymbol{Q} \boldsymbol{q}_{i}+\boldsymbol{q}_{i}^{T} \boldsymbol{g}+C \\
& =\left\|\boldsymbol{Q}^{1 / 2} \boldsymbol{q}_{i}-\boldsymbol{Q}^{-1 / 2} \boldsymbol{g}\right\|^{2}-\left\|\boldsymbol{Q}^{-1 / 2} \boldsymbol{g}\right\|^{2}+C
\end{aligned}
$$

TABLE I

DESIGN SPECIFICATIONS AND PARAMETERS FOR SUBFILTERS $q_{i}(z), i=0,1,2$, OF STRUCTURAL PR Two-CHANNEl FIR TRIPLET FILTER BANKS AND WAVELET BASES IN EXAMPLE 1 ([9, EXAMPLE 2(b) ]) AND EXAMPLE 2

\begin{tabular}{c|c|c}
\hline \hline Parameters & Example 1 (Ex. 2(b) in [9]) & Example 2 \\
\hline$\omega_{p 0}=\omega_{p 1}=\omega_{p 2}$ & $0.4 \pi$ & $0.4 \pi$ \\
\hline$L_{q 0}, L_{q 1}, L_{q 2}$ & $6,14,14$ & $6,14,14$ \\
\hline$N_{0}, N_{1}, N_{2}$ & $3,9,13$ & $3,7,9$ \\
\hline$K_{q 0}, K_{q 1}, K_{q 2}$ & $0,0,0$ & $3,3,3$ \\
\hline \hline
\end{tabular}

where $\Omega$ is the spectral support over which $q_{i}^{(d)}\left(e^{j \omega}\right)$ is to be approximated

$$
\begin{aligned}
{[\boldsymbol{Q}]_{n, m} } & =\int_{\Omega} W^{2}(\omega) \cdot e^{j(m-n) \omega} d \omega \\
{[\boldsymbol{g}]_{n} } & =\int_{\Omega} W^{2}(\omega) \cdot \operatorname{Re}\left\{q_{i}^{(d)}\left(e^{j \omega}\right) \cdot e^{j n \omega}\right\} d \omega \\
C & =\int_{\Omega}\left|W(\omega) \cdot q_{i}^{(d)}\left(e^{j \omega}\right)\right|^{2} d \omega .
\end{aligned}
$$

This is equivalent to

$\min _{\boldsymbol{q}_{i}} \delta_{i}$ subject to $\delta_{i}$

$$
-\left\|\boldsymbol{Q}^{1 / 2} \boldsymbol{q}_{i}-\boldsymbol{Q}^{-1 / 2} \boldsymbol{g}\right\|^{2}+\left\|\boldsymbol{Q}^{-1 / 2} \boldsymbol{g}\right\|^{2}-C \geq 0 .
$$

To solve (2.18a) using SDP, Schur complement allows us to rewrite it as

$$
\min _{\boldsymbol{q}_{i}} \delta_{i} \text { subject to } \boldsymbol{\Psi}\left(\boldsymbol{q}_{i}\right) \geq 0
$$

where

$\boldsymbol{\Psi}\left(\boldsymbol{q}_{i}\right)=\left(\begin{array}{cc}\delta_{i}+\left\|\boldsymbol{Q}^{-1 / 2} \boldsymbol{g}\right\|^{2}-C & \boldsymbol{q}_{i}^{T} \boldsymbol{Q}^{1 / 2}-\left(\boldsymbol{Q}^{-1 / 2} \boldsymbol{g}\right)^{T} \\ \boldsymbol{Q}^{1 / 2} \boldsymbol{q}_{i}-\boldsymbol{Q}^{-1 / 2} \boldsymbol{g} & \boldsymbol{I}\end{array}\right)$.

The advantage of formulating the objective function as a set of LMI is that other linear equalities, inequalities, and convex quadratic constraints can easily be incorporated. This is illustrated in the following by incorporating the peak stopband ripple constraints into the analysis filter pair. Let $\delta_{p, m}$ be the peak stopband ripple of $H_{m}(z), m=0,1$, such that

$$
\left|H_{m}\left(e^{j \omega}\right)\right| \leq \delta_{p, m}, \text { for } \omega \text { in the stopband. }
$$

Given $q_{0}(z)$ and substituting (2.3a) into (2.19), we have the following convex quadratic inequality constraint for incorporating the peak constraint into $H_{0}(z)$ :

$$
\beta_{R, 0}^{2}(\omega)+\beta_{I, 0}^{2}(\omega) \leq \delta_{p, 0}^{2}, \text { for } \omega \in\left[\pi-\omega_{p 1}, \pi\right]
$$

where

$$
\begin{aligned}
\beta_{R, 0}(\omega)= & \mid \frac{1}{2} \boldsymbol{q}_{1}^{T} \boldsymbol{c}_{1} \cdot \operatorname{Re}\left\{e^{-j 2 N_{0} \omega}-p \cdot e^{-j \omega} q_{0}\left(e^{j 2 \omega}\right)\right\} \\
& +\frac{1}{2}(1+p) \cos \left(\left(2 N_{1}+1\right) \omega\right) \mid \\
\beta_{I, 0}(\omega)= & \mid \frac{1}{2} \boldsymbol{q}_{1}^{T} \boldsymbol{s}_{1} \cdot \operatorname{Im}\left\{e^{-j 2 N_{0} \omega}-p \cdot e^{-j \omega} q_{0}\left(e^{j 2 \omega}\right)\right\} \\
& -\frac{1}{2}(1+p) \sin \left(\left(2 N_{1}+1\right) \omega\right) \mid .
\end{aligned}
$$




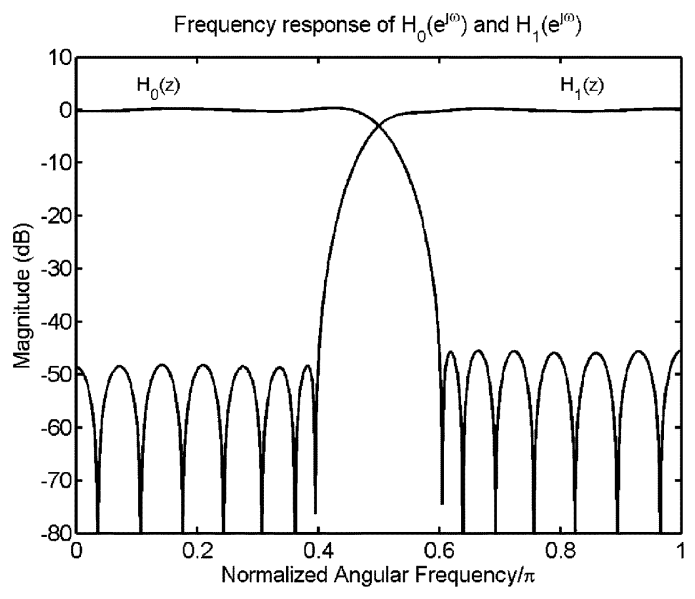

(a)

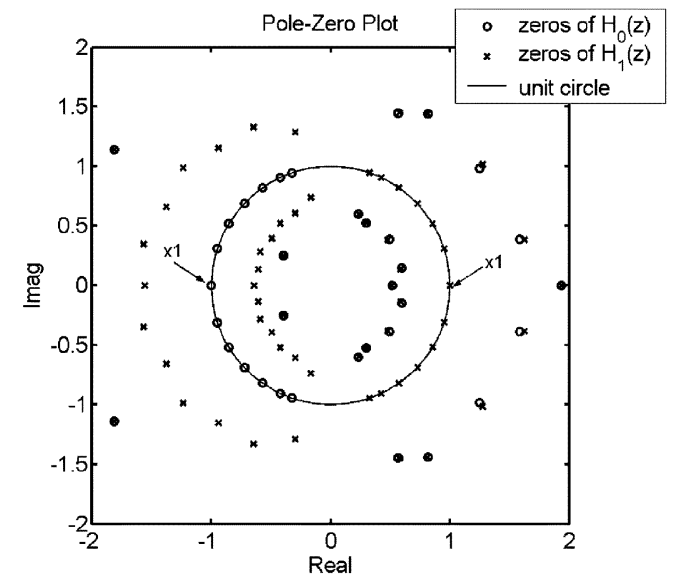

(c)

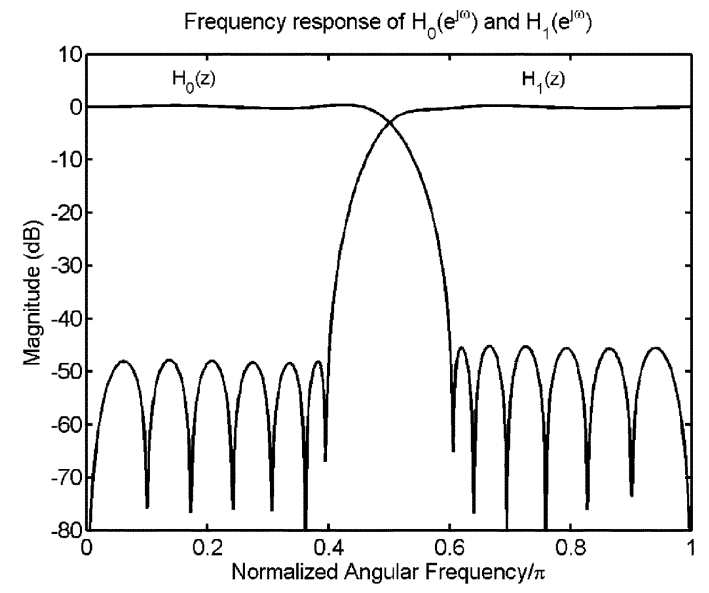

(b)

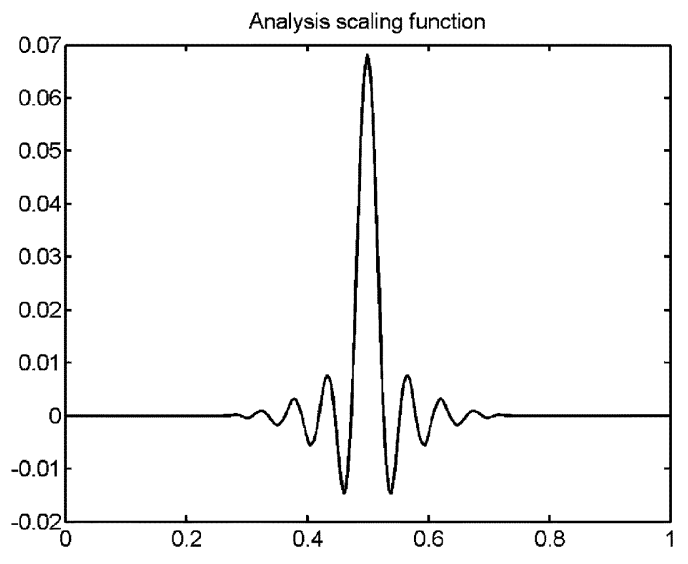

(d)

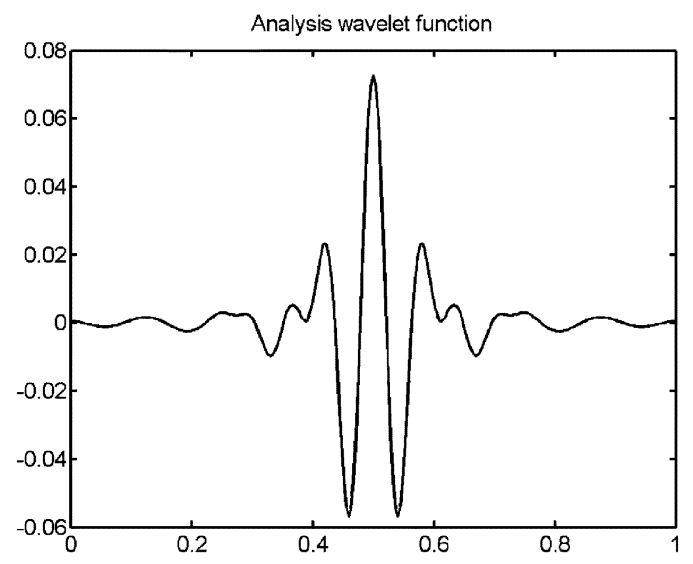

(e)

Fig. 3. (a) Frequency response of the analysis filter pair $H_{m}(z), m=0,1$, in Example 1. (b) Frequency response. (c) Plot-zero plot. (d) Analysis scaling function. (e) Analysis wavelet function of the linear-phase wavelet FB in Example 1. One zero is imposed, respectively, at $z=-1$ and 1 on $H_{0}(z)$ and $H_{1}(z)$.

TABLE II

Design Summary of Analysis Filter Pair $H_{m}(z), m=0,1$, of Structural PR Two-Channel FIR Triplet FBs AND

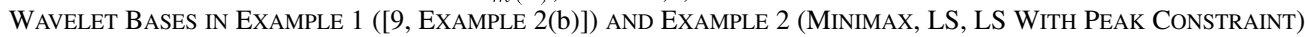

\begin{tabular}{l|c|c}
\hline \hline & Example 1 (Ex. 2(b) in [9]) & Example 2 \\
\hline Group delay of $H_{0}(z), H_{1}(z)$ & 19,32 & 15,24 \\
\hline Passband deviation of $H_{0}(z) / \mathrm{dB}$ & $0.2697(0.2702)$ & $(0.4158,0.6659,0.4877)$ \\
\hline Stopband attenuation of $H_{0}(z) / \mathrm{dB}$ & $45.46(44.73)$ & $(42.22,33.28,40.03)$ \\
\hline Passband deviation of $H_{1}(z) / \mathrm{dB}$ & $0.2706(0.2702)$ & $(0.4365,0.6889,0.5149)$ \\
\hline Stopband attenuation of $H_{1}(z) / \mathrm{dB}$ & $48.15(47.33)$ & $(44.81,36.29,43.05)$ \\
\hline \hline
\end{tabular}


Similarly, the peak constraint of $H_{1}(z)$ is given by

$$
\beta_{R, 1}^{2}(\omega)+\beta_{I, 1}^{2}(\omega) \leq \delta_{p, 1}^{2} \text { for } \omega \in\left[0, \omega_{p 2}\right]
$$

where

$$
\begin{aligned}
\beta_{R, 1}(\omega)= & \mid \frac{p-1}{1+p} \boldsymbol{q}_{2}^{T} \boldsymbol{c}_{2} \cdot \operatorname{Re}\left\{H_{0}\left(e^{j \omega}\right)\right\} \\
& +\operatorname{Re}\left\{\frac{1}{1+p} e^{-j 2 N_{2} \omega}\left[e^{-j 2 N_{0} \omega}-p \cdot e^{-j \omega} q_{0}\left(e^{j 2 \omega}\right)\right]\right\} \mid \\
\beta_{I, 1}(\omega)= & \mid \frac{p-1}{1+p} \boldsymbol{q}_{2}^{T} \boldsymbol{s}_{2} \cdot \operatorname{Im}\left\{H_{0}\left(e^{j \omega}\right)\right\} \\
& +\operatorname{Im}\left\{\frac{1}{1+p} e^{-j 2 N_{2} \omega}\left[e^{-j 2 N_{0} \omega}-p \cdot e^{-j \omega} q_{0}\left(e^{j 2 \omega}\right)\right]\right\} \mid .
\end{aligned}
$$

Similar to the minimax case, we densely discretize $\omega$ over the stopband region into a set of frequency points $\omega_{k}, k=1, \ldots, K$. This yields the following form for (2.20a) and (2.20b):

$$
\Lambda_{k}\left(\boldsymbol{q}_{m+1}\right)=\left(\begin{array}{ccc}
\delta_{p, m}^{2} & \beta_{R, m}\left(\omega_{k}\right) & \beta_{I, m}\left(\omega_{k}\right) \\
\beta_{R, m}\left(\omega_{k}\right) & 1 & 0 \\
\beta_{I, m}\left(\omega_{k}\right) & 0 & 1
\end{array}\right) \geq 0 .
$$

These constraints on the peak ripples can be augmented to the existing LMI for LS criterion in (2.18b). An alternative approach to impose the peak constraint can be achieved by adjusting the weighting function in (2.17). However, this will require an iterative method to determine the exact ripples at the target frequency bands. To incorporate the $K$ regularity conditions mentioned in Section II-B, we can substitute (2.15) into (2.18b) for the LS criterion and (2.21) for the LS criterion with peak error constraints. By defining the augmented variable $\boldsymbol{x}_{i}^{T}=\left[\begin{array}{ll}\delta_{i} & \boldsymbol{q}_{i, L_{q i}-r_{i}}^{T}\end{array}\right]$, all the constraints can be stacked together to form a standard SDP problem.

\section{Design Examples}

In all the design examples, the constant parameter $p$ was chosen as $\sqrt{2}-1$. By so doing, the magnitudes of the analysis filter pair $\left(H_{0}(z)\right.$ and $\left.H_{1}(z)\right)$ at $\omega=\pi / 2$ are both equal to $1 / \sqrt{2}$. As a result, a more symmetric frequency response can be obtained. Unless otherwise specified, the minimax error criterion is employed so that the frequency response in the stopband region is equiripple. The weighting function is chosen as 1 for both passband and stopband. The frequency variable $\omega$ in the band of interest was uniformly discretized using $K=500$ samples. The SDP optimization was then carried out using the MATLAB LMI Toolbox and it took less than 60 iterations and less than $1 \mathrm{~min}$ to obtain the solution in a Pentium III-866 personal computer.

Example 1-Structural PR Two-Channel Linear-Phase FIR Triplet FBs and Wavelet Bases: For comparison purposes, a structural PR two-channel linear-phase FIR triplet FB with the same specifications as [9, Ex. 2(b)] was designed using the proposed SDP method. The parameters of the subfilters $q_{i}(z)$ are shown in the second column of Table I. The frequency responses of the low-pass and high-pass analysis filters are shown in Fig. 3(a). It should be noted that there is no regularity in this triplet FB. The results and performances of the analysis filter pair are summarized in the second column of Table II. It can be seen that the passband and stopband errors of the analysis
TABLE III

DESIGN SPECIFICATIONS AND PARAMETERS FOR SUBFILTERS $q_{i}(z), i=0,1,2$ OF Two-LEVEL TREE-STRUCTURED FOUR-CHANNEL LOW-DELAY TRIPLET-BASED WAVELET BASES IN EXAMPLE 3

\begin{tabular}{c|c|c}
\hline \hline Parameters & Level 1 & Level 2 \\
\hline$\omega_{p 0}^{(l)}=\omega_{p 1}^{(l)}=\omega_{p 2}^{(l)}$ & $0.45 \pi$ & $0.4 \pi$ \\
\hline$L_{q 0}^{(l)}, L_{q 1}^{(l)}, L_{q 2}^{(l)}$ & $20,24,22$ & $10,14,14$ \\
\hline$N_{0}^{(l)}, N_{1}^{(l)}, N_{2}^{(l)}$ & $8,16,15$ & $4,8,9$ \\
\hline$K_{q 0}^{(l)}=K_{q 1}^{(l)}=K_{q 2}^{(l)}$ & 4 & 4 \\
\hline \hline
\end{tabular}

filter pair using the proposed SDP method are comparable to that reported in [9].

Next, we consider a wavelet FB with the same parameters except the parameters $K_{q 0}, K_{q 1}, K_{q 2}$ are equal to one (as mentioned earlier, this problem has not been considered in [9], due to the difficulties in imposing the regularity constraint). In other words, the low-pass and high-pass analysis filters have one zero at $z=-1$ and 1, respectively. Fig. 3(b) and (c) shows the frequency response and the pole-zero plot of the analysis filters. It can be seen that the prescribed zeros are properly imposed. The analysis scaling and wavelet functions so obtained are shown in Fig. 3(d) and (e), respectively. This demonstrates the effectiveness of the proposed SDP method in imposing the $K$ regularity conditions.

Example 2-Structural PR Two-Channel Low-Delay FIR Triplet Wavelet Bases: In this example, a structural PR two-channel low-delay FIR triplet wavelet with prescribed order of $K$ regularity was designed. The subfilter lengths $L_{q i}$ and cutoff frequencies $\omega_{p i}$ of $q_{i}(z)$ are identical to Example 1 except that the system delay is now reduced from 51 samples in the linear-phase case to 39 samples. Furthermore, a $K$ regularity of $K_{0}=K_{1}=3$ is imposed. In other words, the FB can be used to construct a wavelet basis. The third column of Table I shows the parameters of the subfilters. The frequency response and group delay of the triplet wavelet FB are respectively shown in Fig. 4(a) and (b). It can be seen that the low-delay triplet wavelet FB is approximately linear-phase in the passband. The regularity of the analysis filter pair, $K_{0}=K_{1}=3$, was verified from the pole-zero plot of $H_{0}(z)$ and $H_{1}(z)$. Due to page limitation, the details are omitted. Fig. 4(c) and (d) shows the analysis scaling and wavelet functions, respectively. They are rather smooth, but not symmetric due to the low-delay constraint. To demonstrate the flexibility of the SDP formulation, the design was again performed using the LS and LS with peak constraints (LSPC) criteria. Fig. 4(e) shows the frequency response of the analysis filter pair when the LS error criterion is employed. A similar triplet FB with peak constraints of $\left|20 \log \left(\delta_{p, 0}\right)\right|=40 \mathrm{~dB}$ and $\left|20 \log \left(\delta_{p, 1}\right)\right|=43 \mathrm{~dB}$ for, respectively, the low-pass and high-pass analysis filters was designed as shown in Fig. 4(f). The performances of the analysis filter pair $H_{m}(z)$ using minimax, LS and LSPC are summarized in the third column of Table III. It can be seen that the passband and stopband ripple errors for the minimax case are slightly increased, as compared with Example 1, in exchange of a lower system delay and the $K$ regularity constraints. Next, we shall consider the construction of a class of tree-structured $M$-channel uniform FBs and wavelets by cascading the two-channel triplet wavelet FBs. 


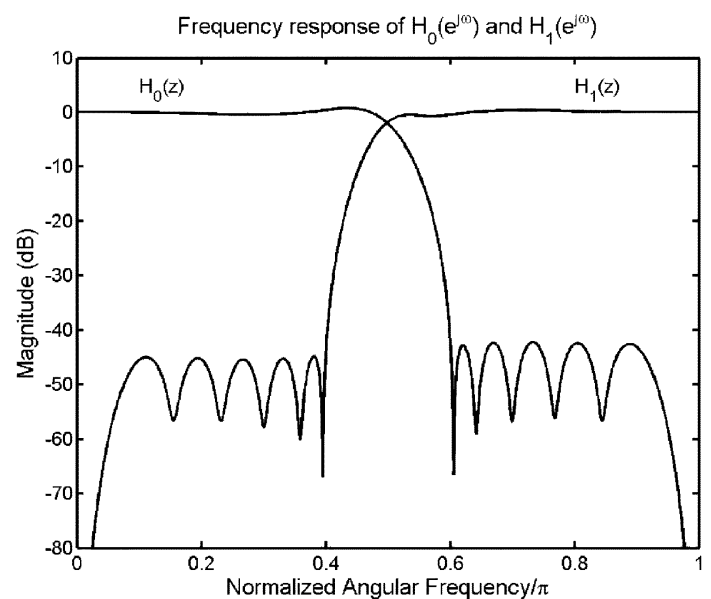

(a)

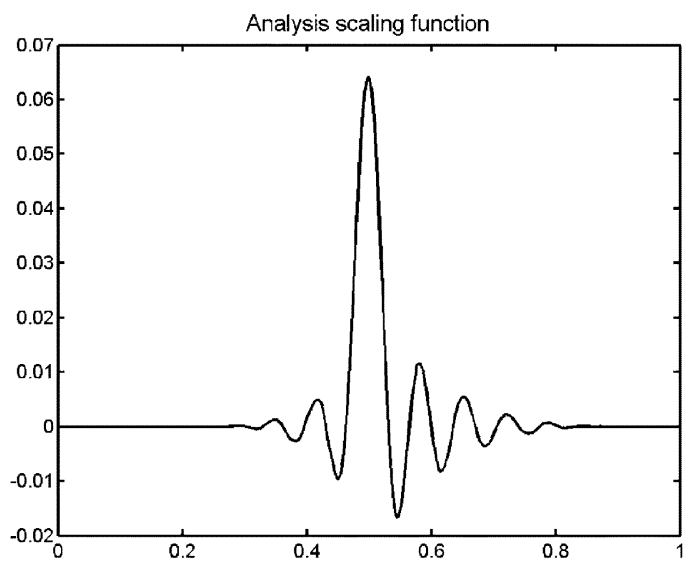

(c)

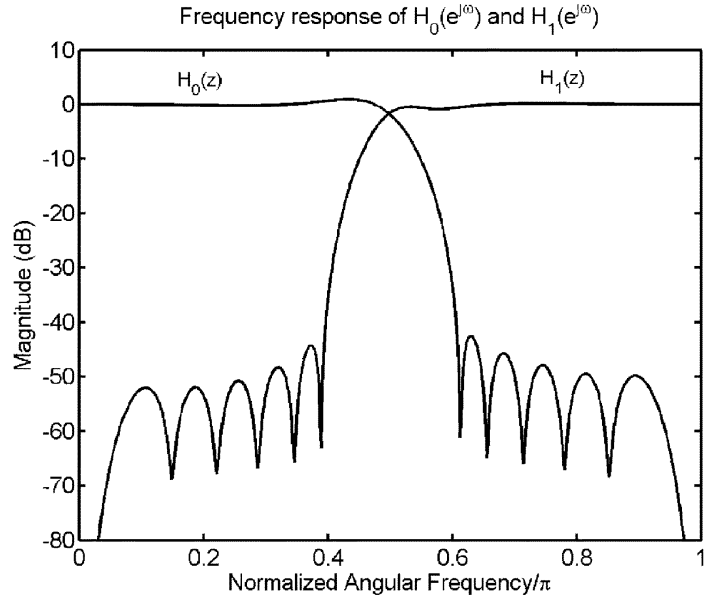

(e)

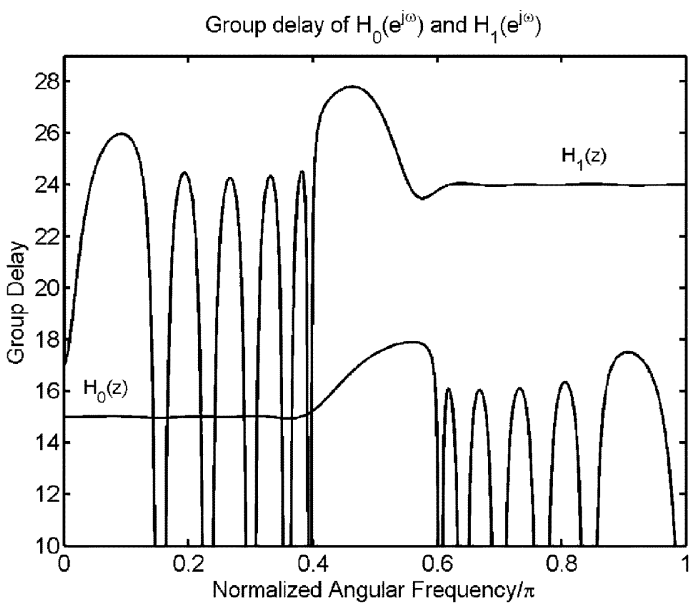

(b)

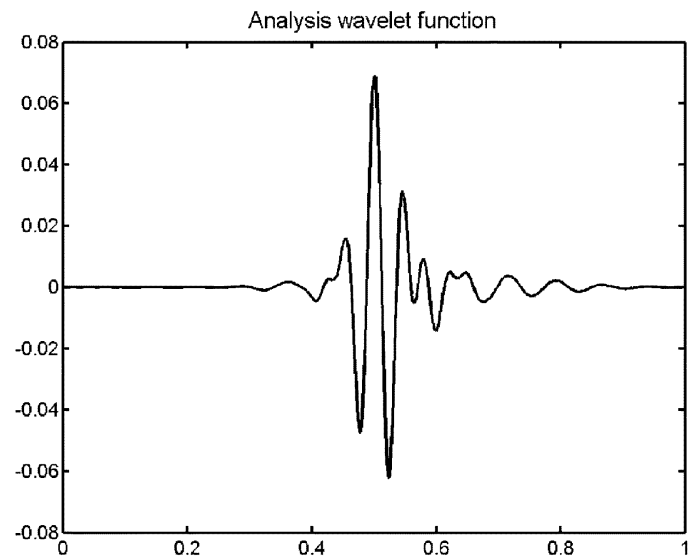

(d)

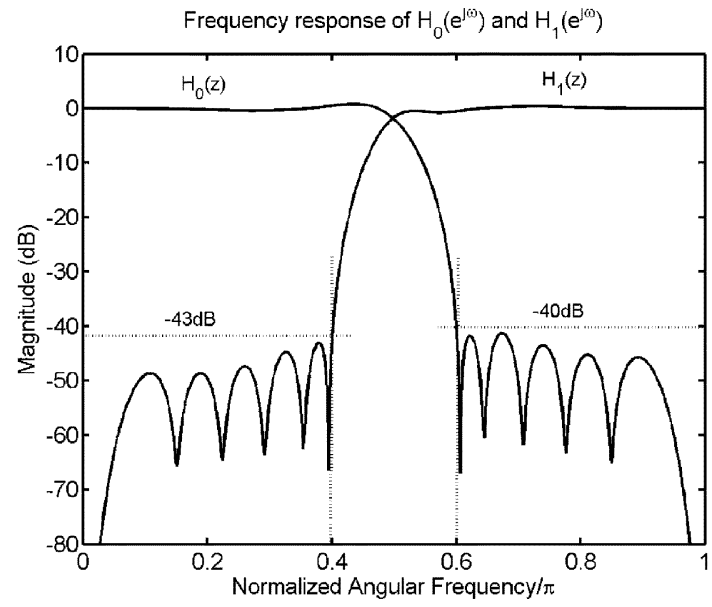

(f)

Fig. 4. Design results of the low-delay triplet FB in Example 2. (a) Frequency response and (b) group delay of the analysis filter pair $H_{m}(z), m=0,1$ using the minimax error criteria. (c) Analysis scaling function. (d) Analysis wavelet function. Frequency responses of the analysis filter pair using (e) the LS error criteria and (f) LS with peak constraints. $K_{0}=K_{1}=3$.

\section{TRIPLET-BASED TREe-STRUCTURED $M$-CHANNEL UNIFORM FBs}

\section{A. Tree-Structured Uniform M-Channel FBs and Wavelet Bases}

In this section, we exploit a type of tree-structured FB, which when combined with the triplet FBs, closely resembles the frequency response of an $M$-channel FB with $M=2^{L}$. There are, of course, many other variations in choosing the tree decomposition to approximate a desired time-frequency resolution. Here, we are particularly interested in approximating a uniform linearphase or passband linear-phase $M$-channel FBs, which usually involve nonlinear constrained optimization with large number of variables. It is shown in the following that by using the triplet FBs and a particular tree-structure, the design complexity can be greatly reduced. More precisely, the triplet FBs are cascaded in a 


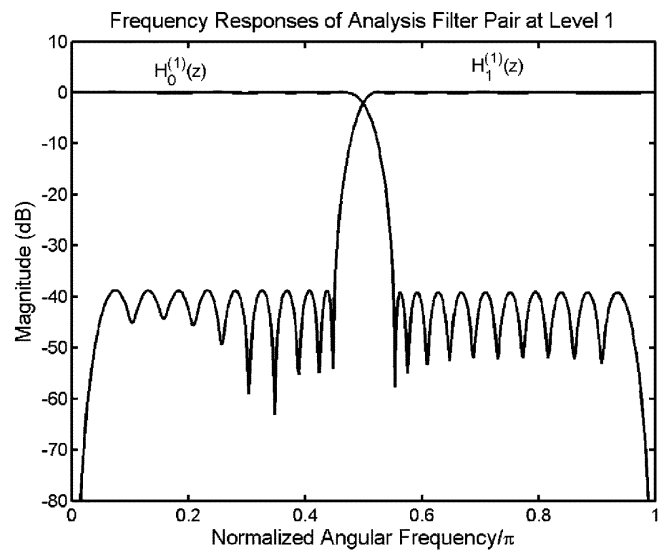

(a)

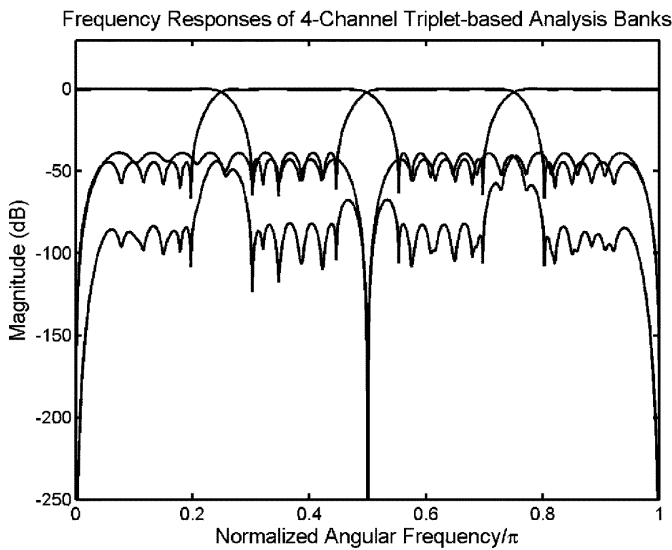

(c)

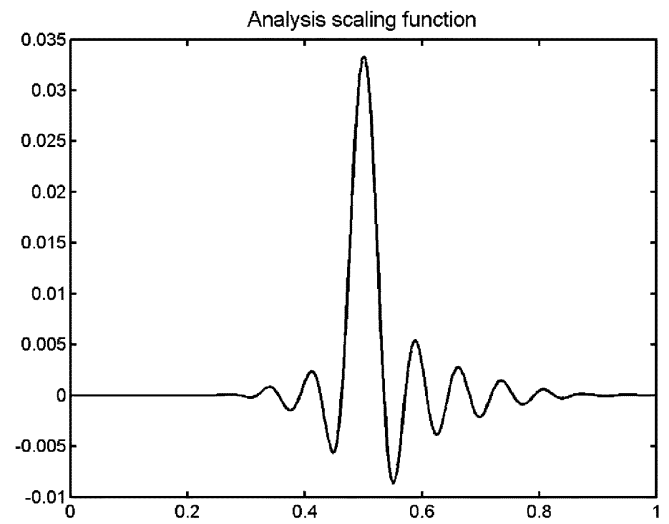

(e)

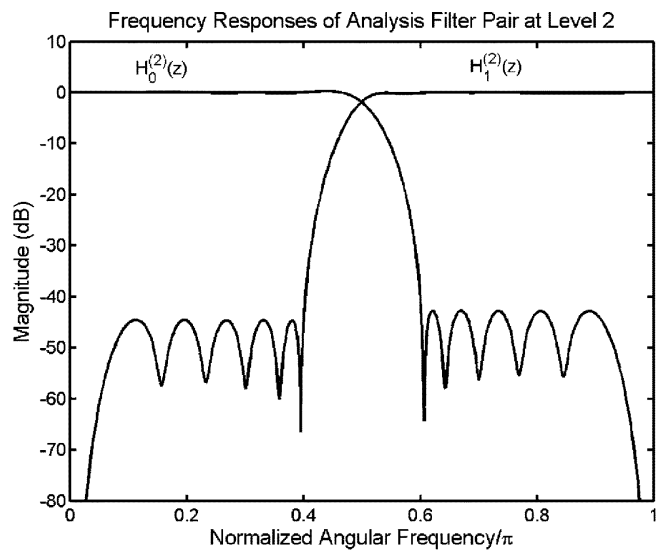

(b)

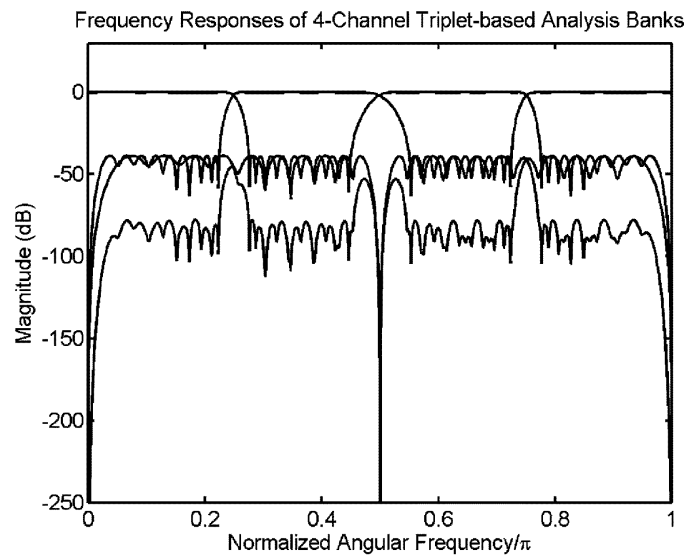

(d)

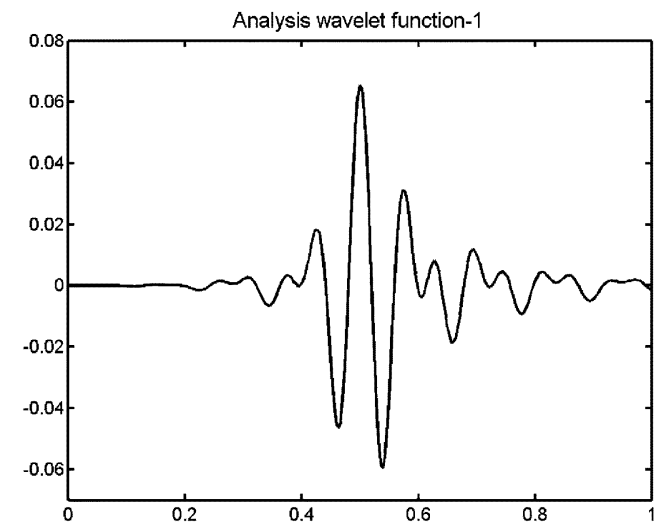

(f)

Fig. 5. Design results of the low-delay tree-structured four-channel FB in Example 3. Frequency responses of the analysis filter pair $H_{m}^{(l)}(z), m=0,1$, at (a) level 1and (b) level 2 of the tree. Frequency response of the four-channel analysis banks using (c) Fig. 5(a) and (b) for level 1 and 2 , respectively, and (d) Fig. 5(a) for all levels of the tree. (e) Analysis scaling function. (f) Analysis wavelet function 1.

tree structure as shown in Fig. 1(b). Instead of using an identical FB at each level, a different triplet FB pair: $H_{m}^{(l)}(z), m=0,1$, is used at the $l$ th level or stage of the tree, where $l=1,2, \ldots, L$. The reason will become apparent later. From the noble identity [14], the tree-structured FB can be redrawn as an $M$-channel FB as shown in Fig. 1(a) by moving $H_{m}^{(l)}(z)$ to the left hand side of the decimators in the tree structure. This gives rise to $M$ analysis filters $H_{m}(z), m=0,1, \ldots, M-1$. For notation convenience, let us treat the index " $l$ " in $H_{m}^{(l)}(z)$ as the $l$ th digits in a weighted binary representation and denote it by $b_{l}$. The equivalent transfer function obtained by passing the signal through the branch $H_{b_{1}}^{(1)}(z), \ldots, H_{b_{L}}^{(L)}(z)$ can then be labeled as $H_{m}(z)$, where $m=b_{L}+2 b_{L-1}+\cdots+2^{L-1} b_{1}=\left(b_{1}, \ldots, b_{L}\right)_{2}$. Then, the final analysis filters $H_{m}(z)$ can be written as

$$
H_{m}(z)=H_{b_{1}}^{(1)}(z) H_{b_{2}}^{(2)}\left(z^{2}\right) \ldots H_{b_{L}}^{(L)}\left(z^{2^{L-1}}\right) .
$$

A similar equation can be derived for the final synthesis filters $F_{m}(z)$. It is clear that the whole system using the tree structure preserves the PR and linear-phase properties if all the twochannel FBs in the tree are also PR and linear phase. Besides, if all the two-channel FBs are wavelet FBs, then, the $M$-channel 
TABLE IV

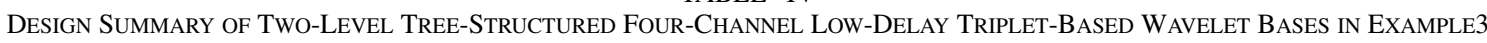

\begin{tabular}{l|c|c}
\hline \hline & Level 1 & Level 2 \\
\hline Transition Bandwidth $\Delta \omega^{(l)}$ & $0.1 \pi$ & $0.2 \pi$ \\
\hline Group Delay of $H_{0}^{(l)}(z), H_{1}^{(l)}(z)$ & 33,46 & 17,26 \\
\hline Passband deviation (Stopband attenuation) of $H_{0}^{(l)}(z) / \mathrm{dB}$ & $0.0732(39.09)$ & $0.0601(42.77)$ \\
\hline Passband deviation (Stopband attenuation) of $H_{1}^{(l)}(z) / \mathrm{dB}$ & $0.0735(38.75)$ & $0.0636(44.54)$ \\
\hline
\end{tabular}

TABLE V

Design Specifications And Parameters for Subfilters $q_{i}^{(l)}(z), i=0,1,2$, of FiVe-LeVel Tree-Structured 32-Channel LINEAR-PHASE TRIPLET-BASED WAVELE

\begin{tabular}{c|c|c|c|c|c}
\hline Parameters & Level 1 & Level 2 & Level 3 & Level 4 & Level 5 \\
\hline$\omega_{p 0}^{(l)}=\omega_{p 1}^{(l)}=\omega_{p 2}^{(l)}$ & $0.4875 \pi$ & $0.475 \pi$ & $0.45 \pi$ & $0.4 \pi$ & $0.3 \pi$ \\
\hline$L_{q 0}^{(l)}, L_{q 1}^{(l)}, L_{q 2}^{(l)}$ & $46,86,78$ & $30,44,40$ & $18,24,22$ & $10,14,14$ & $8,8,8$ \\
\hline$N_{0}^{(l)}, N_{1}^{(l)}, N_{2}^{(l)}$ & $23,65,81$ & $15,36,41$ & $9,20,22$ & $5,11,13$ & $4,7,7$ \\
\hline$K_{q 0}^{(l)}=K_{q 1}^{(l)}=K_{q 2}^{(l)}$ & 5 & 5 & 5 & 5 & 5 \\
\hline
\end{tabular}

FB is also a wavelet FB. Next, we consider the choice of transition bandwidths and filter length of the two-channel FBs. Suppose that identical two-channel FBs are employed at all levels in the tree. Then, the transition bandwidth of $H_{m}(z)$ will not be identical due to the upsampling of $z$ in moving $H_{b_{l}}^{(l)}(z)$ to the left hand side of the decimators. As a result, higher implementation complexity is required to achieve a given transition bandwidth and stopband attenuation of the $M$-channel FB. This is illustrated in Fig. 5(d) by cascading the FB in Fig. 5(a) to form a tree structure of two levels. The resulting four-channel FB does not have a constant transition bandwidth. This also significantly increases the total system delay (237 samples in the above example). To avoid this problem, it is therefore advantageous to properly widen the transition bandwidth of the two-channel FB as the level increases so that an identical transition bandwidth can be achieved in the final $M$-channel FB [15], [17], [18]. More precisely, due to the upsampling by a factor of $2^{l-1}$ at the $l$ th level, the transition bandwidth of $H_{b_{l}}^{(l)}\left(z^{2^{l-1}}\right)$ will be $2^{l-1}$ times narrower than that of $H_{b_{l}}^{(l)}(z)$. To achieve a uniform transition bandwidth, the transition bandwidth of $H_{b_{l}}^{(l)}(z)$ should satisfy

$$
\Delta \omega^{(1)}=\frac{\Delta \omega^{(2)}}{2}=\cdots=\frac{\Delta \omega^{(L)}}{2^{L-1}}
$$

where $\Delta \omega^{(l)}$ is the transition bandwidth of the analysis filter pair at the $l$ th level. It can be seen that the deeper the level, the wider will be the transition bandwidth. For a given stopband attenuation, the filter lengths of the two-channel FBs in the tree can therefore be reduced approximately by a factor of two when the level of the tree increases. This considerably reduces the total system delay and the hardware complexity of the tree-structured $M$-channel FB. Using the triplet FBs and the SDP approach, the design and implementation complexities can be considerably reduced. For a given transition bandwidth of the $M$-channel uniform FB, the transition bandwidths of the triplet FBs in each level of the tree can be determined according to (3.2). A triplet FB satisfying the given stopband attenuation and transition bandwidth at the first level is first designed. Let the subfilter lengths of the subfilters $q_{i}^{(1)}(z)$ at the first level be $L_{q i}^{(1)}$. Then, the subfilter lengths of $q_{i}^{(l)}(z)$ at the $l$ th level are approximately $L_{q i}^{(1)} / 2^{l-1}$. They can be independently designed by the SDP method and cascaded to form the final $M$-channel FB. For $M=2^{L}$ channels, only $L$ different two-channel triplet FBs need to be designed separately using the SDP method. Due to this nice property, the proposed method serves as a very attractive alternative for designing uniform FB with very large number of channels, which usually involves constrained nonlinear optimization with a large number of variables, causing considerable difficulties in converging to the optimal solution. In addition, the PR and regularity conditions are structurally imposed and the final FB can be realized efficiently using SOPOT coefficients, as we shall see later in Section IV. We now consider two design examples.

\section{B. Design Examples}

Example 3-Structural PR Four-Channel Low-Delay Triplet-Based Wavelet Bases: A structural PR uniform four-channel low-delay triplet-based wavelet bases was designed by cascading two levels of two-channel low-delay FIR triplet wavelet FB. They are independently designed using the proposed SDP method. The specifications and parameters of the subfilters $q_{i}^{(l)}(z)$ at all levels are summarized in Table III. The frequency responses of $H_{b_{l}}^{(l)}(z), l=1,2$, so obtained are shown in Fig. 5(a) and (b), respectively. Fig. 5(c) shows the final analysis banks of the four-channel wavelet FB. It can be seen that a uniform transition bandwidth of $\Delta \omega^{(1)}=0.1 \pi$ is achieved by properly widening the transition bandwidths of the two-channel FBs as the level of the tree increases. This four-channel triplet wavelet FB has a $K$ regularity of fourth order and its stopband attenuation and system delay are $40 \mathrm{~dB}$ and 165 samples, respectively. It will be increased to $225 \mathrm{sam}-$ ples if linear-phase triplet wavelets are employed to achieve the same stopband attenuation. The design results are summarized in Table IV. Fig. 5(e) and (f) shows the analysis scaling and the first wavelet functions of the four-channel triplet wavelets (other wavelet functions are not shown here). They are rather smooth, but not symmetric due to the low-delay constraint. The design time is 123 seconds for the whole system. Next, we shall 


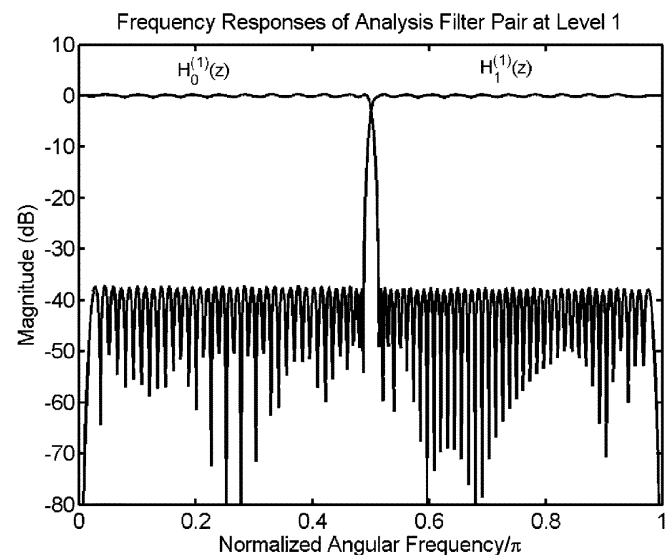

(a)

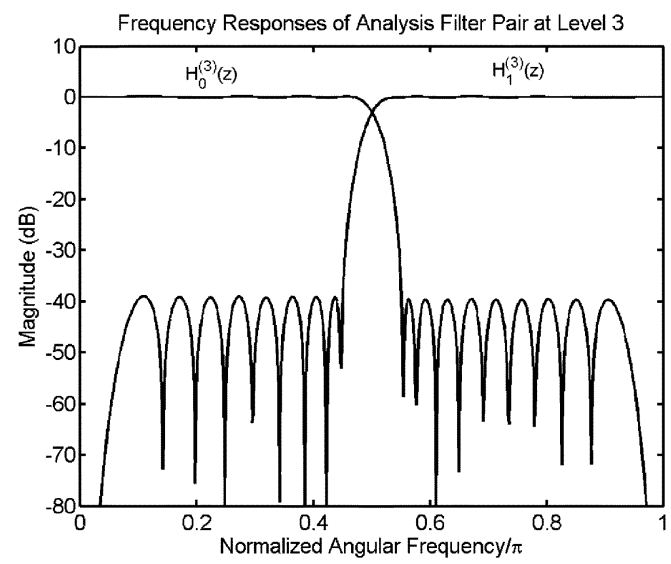

(c)

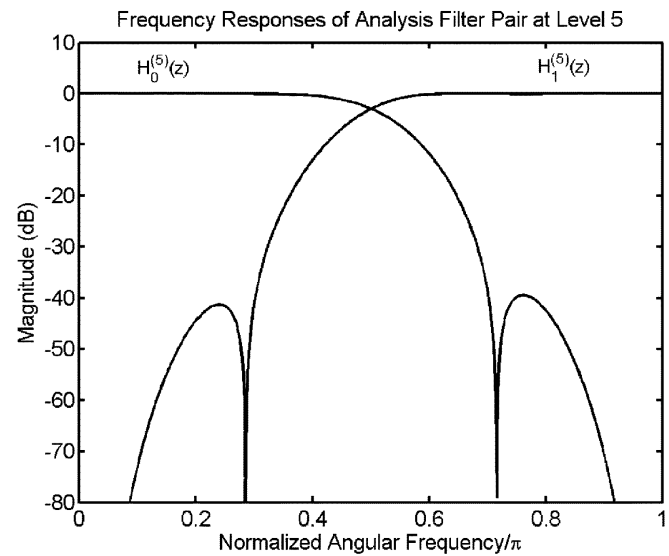

(e)

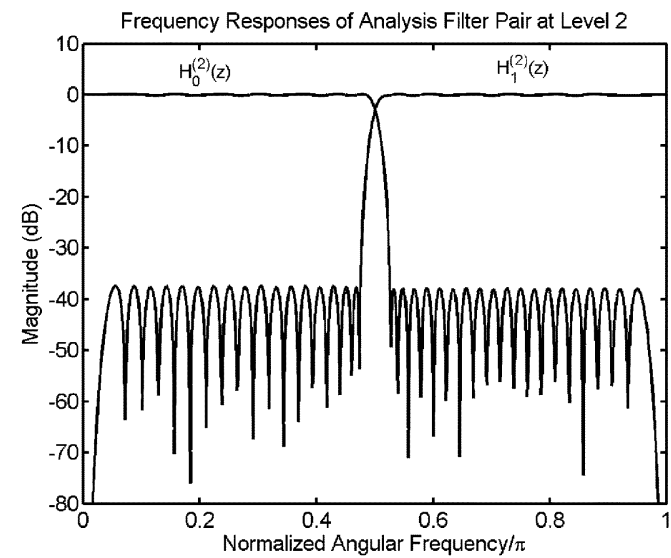

(b)

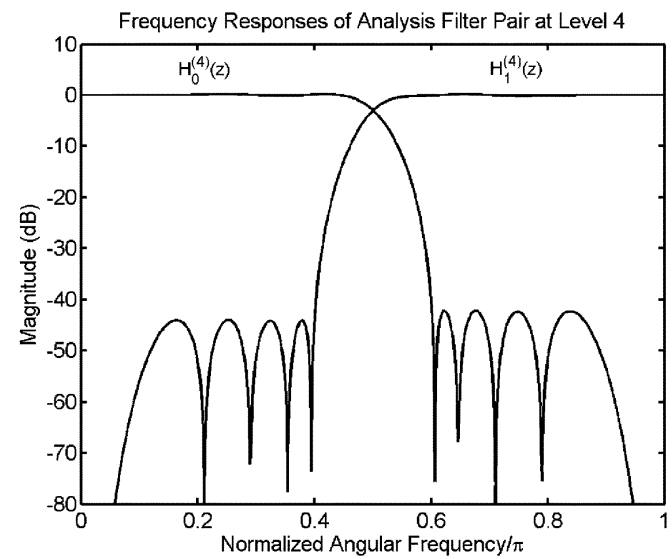

(d)

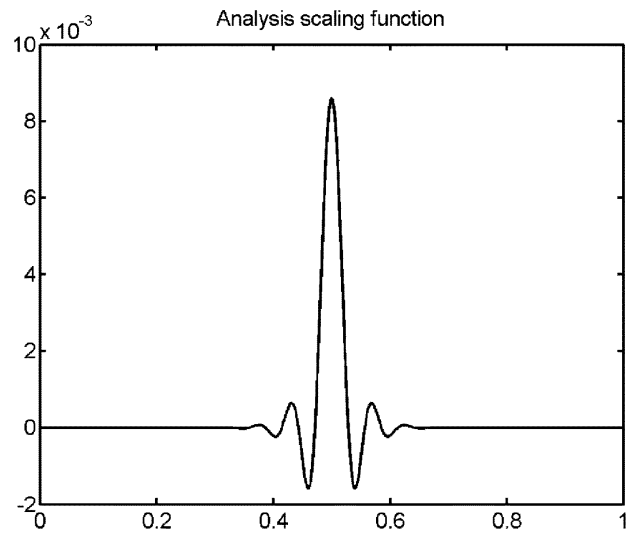

(f)

Fig. 6. Design results of the 32-channel linear-phase wavelet FB in Example 4. (a)-(e) Frequency responses of the analysis filter pair at level 1-5, respectively. (f) Analysis scaling function.

consider a triplet-based tree-structured $M$-channel FBs with larger number of channels.

Example 4-Structural PR 32-Channel Linear-Phase Triplet-Based Wavelet Bases: In this example, a structural PR 32-channel uniform linear-phase triplet-based wavelet bases was constructed by cascading five levels of two-channel linear-phase FIR triplet wavelet FB in a tree structure. Table V summarizes the parameters of the subfilters $q_{i}^{(l)}(z)$. The frequency responses of $H_{b_{l}}^{(l)}(z), l=1,2,3,4,5$, so obtained are shown in Fig. 6(a)-(e), respectively. The 32-channel analysis banks are shown in Fig. 7, which has a $K$ regularity of the fifth order. The transition bandwidth and the stopband attenuation are respectively $\Delta \omega^{(1)}=0.025 \pi$ and around $40 \mathrm{~dB}$. The design results are summarized in Table VI. The total system delay is 2185 samples. If identical two-channel triplet wavelet FB (at level 1) are employed for all levels, the total system delay will be drastically increased to 10509 samples. Moreover, the implementation complexity will be significantly increased. Fig. 6(f) shows the analysis scaling function of the 32-channel triplet-based wavelets. Due to page limitation, details of the wavelet functions are omitted. They are very smooth and unlike those in [12], all of them are symmetric. The design time is 533 


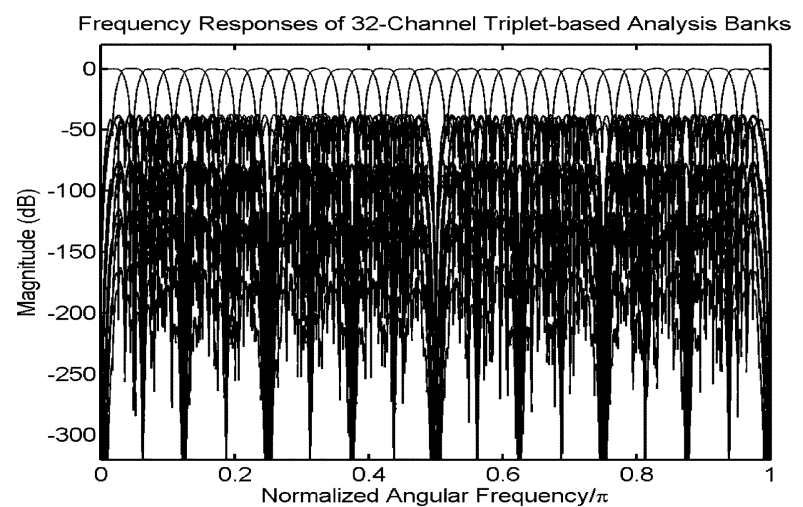

Fig. 7. Frequency response of the 32-channel linear-phase wavelet FB in Example 4.

$\mathrm{s}$ for the whole system. It is clear that uniform FBs with even larger number of channels, say 256, can be designed without much difficulty. Due to page limitation, design results for a similar triplet-based 32-channel FB with a lower system delay using the low-delay two-channel triplet FB is omitted.

\section{Multiplierless TRIPLET-BASED FIR FBS AND WAVELETS}

In this section, we shall study the multiplierless realization of the two-channel triplet wavelet FB. In practical realization of the triplet-based wavelet FBs, the subfilter coefficients of $q_{i}(z), i=0,1,2$, have to be quantized, and the $K$ regularity conditions might no longer hold. Here, we adopt a new realization technique that we have recently proposed in [30] for preserving the $K$ regularity conditions of the two-channel structural PR FIR FB [1] when the filter coefficients are expressed as SOPOT coefficients of the form: $\hat{q}_{i, n}=\sum_{k=1}^{N} a_{k} \cdot 2^{-b_{k}}$, where $a_{k} \in\{-1,0,1\}$ and $b_{k} \in\left\{-l_{b}, \ldots .1,0, \ldots u_{b}\right\}$. The larger the numbers $l_{b}, u_{b}$, and $N$, the closer the SOPOT approximation will be to the original real number. In particular, the method in [30] is used to enforce the necessary zeros for $q_{i}(z)$ in (2.10), even when their coefficients are quantized to SOPOT coefficients. Unfortunately, it was found that because of the inter-relationship of $p_{i}, i=0,1,2$, the $K$ regularity condition can only be achieved exactly when they are real numbers. In practical implementation, their wordlengths have to be long enough so that the error generated will be well below the round-off noise. In design example 5 to be presented, a wordlength of 16 bits is found to yield a good approximation up to $-120 \mathrm{~dB}$. As mentioned earlier in Section II-B, the $K$ regularity of $H_{0}(z)$ and $F_{0}(z)$ are respectively $K_{0} \geq \min \left\{K_{q 0}, K_{q 1}\right\}$ and $K_{1} \geq$ $\min \left\{K_{q 0}, K_{q 1}, K_{q 2}\right\}$, where $K_{q i}$ is the number of zeros of the halfband filter $H_{q i}(z)=\left[z^{-1} q_{i}\left(z^{2}\right)+z^{-2 N_{q_{i}}}\right] / 2$ at $z=-1$. To structurally impose the required zeros at $z=-1$, one may express the halfband filters $H_{q i}(z)$ using the Bernstein polynomial expansion. Since the subfilter coefficients of $q_{i}(z)$ are linear combination of the Bernstein coefficients, which will be quantized to SOPOT coefficients, so are the subfilter coefficients. Therefore, it is possible to construct triplet-based wavelet bases with SOPOT coefficients. Note, the triplet FBs are not restricted to be linear phase. Unfortunately, it was found in [30] that this approach usually yields SOPOT coefficients with very long coefficient wordlength. A new realization technique [30] with a much lower coefficient wordlength, which provides multiplierless realization up to fifth order of zero moments, is employed. More precisely, the subfilter $q_{i}(z)$ is written as

$$
q_{i}(z)=R_{q i}(z)+\left(1-z^{-1}\right)^{K_{q i}} Q_{q i}(z)
$$

where $R_{q i}(z)=\sum_{n=0}^{K_{q i}-1} r_{i, n} z^{-n}$ and $Q_{q i}(z)=$ $\sum_{n=0}^{L_{q i}-K_{q i}-1} s_{i, n} z^{-n}$ are respectively the remainder and quotient obtained by dividing $q_{i}(z)$ by $\left(1-z^{-1}\right)^{K_{q i}}$. Substituting (4.1) into (2.6), we have

$$
H_{q i}(z)=\frac{\left[z^{-2 N_{q i}+1}+R_{q i}\left(z^{2}\right)+\left(1-z^{-2}\right)^{K_{q i}} Q_{q i}\left(z^{2}\right)\right]}{2}
$$

from which we see that if $H_{q i}(z)$ contains the factor $\left(1 \pm z^{-1}\right)^{K_{q i}}$, so is $z^{-2 N_{q i}+1}+R_{q i}\left(z^{2}\right)$. This is equivalent to the condition that the halfband filter $\left[z^{-2 N_{q i}+1}+R_{q i}\left(z^{2}\right)\right] / 2$ has maximal flatness at $z=-1$, and the remainder coefficients of $R_{q i}(z)$ are [6]

$$
\begin{array}{r}
r_{i, n}=\frac{2^{-K_{q i}+1}(-1)^{n} \prod_{m=0}^{K_{q i}-1}\left(2 m-2 N_{q i}+1\right)}{\left(2 n-2 N_{q i}+1\right) n !\left(K_{q i}-1-n\right) !} \\
\text { for } n=0,1, \ldots, K_{q i}-1 .
\end{array}
$$

Once (4.3) is satisfied, the quotient coefficients $s_{i, n}$ of $Q_{q i}(z)$ can be quantized to arbitrary accuracy without violating the prescribed $K$ regularity conditions (if $p_{i}$ s are exact). In [6], Pei and Wang proposed another efficient form for implementing such maximally flat halfband filter. Instead of expanding the remainder coefficients about $z^{-2}$, $R_{q i}\left(z^{2}\right)$ is expanded as $R_{q i}\left(z^{2}\right)=\sum_{n=0}^{K_{q i}-1} b_{i, n}\left(z^{-2}-1\right)^{n}$, where the coefficients $b_{i, n}$ is given by: $b_{i, 0}=1$, and $b_{i, n}=\left[\prod_{m=0}^{n-1}\left(2 N_{q i}-1-m\right)\right] / 2^{n} n !, n=1, \ldots, K_{q i}-1$. It can be shown that $b_{i, n}$ and $r_{i, n}$ are related by $r_{i, n}=$ $\sum_{m=0}^{K_{q i}-1-n} n C_{m} \cdot b_{i, n}(-1)^{n-m}$, where $n C_{m}$ is the binomial coefficient. If $b_{i, n}$ can be exactly represented as SOPOT coefficients, then so are the coefficients $r_{i, n}$. This was shown to be true for $n=0,1,2,3,4$ [30]. To obtain a multiplierless PR wavelet FB, the coefficients of $Q_{q i}(z)$ are represented as SOPOT coefficients. To further reduce the number of adders in realizing $Q_{q i}(z)$, they can be implemented in transposed form using the technique of multiplier block (MB) [27]. It is a very useful technique to reduce the number of adders needed to implement the multiplications of an integer input with a set of constant integer coefficients. Therefore, the implementation complexity of the structural PR FB can be drastically reduced. Following [30], the random search algorithm proposed in [25], [30] is employed, because of its simplicity and effectiveness. Due to page limitation, the detail is omitted and interested readers are referred to [25] and [30] for more details. Note, from Fig. 2, the constant parameters $C_{0}$ and $C_{1}$ are not implemented in practice since the product of $C_{0} C_{1}$ is exactly equal to 0.5 . It can be done by shifting the signal by one bit to the least-significant-bit (LSB) direction. However, for $p_{0}=-p$, $p_{1}=1 /(1+p), p_{2}=\left(p^{2}-1\right) / 2$ and $p=\sqrt{2}-1$, there is no SOPOT approximation of $p$ with finite number of terms such 
TABLE VI

Design Summary of Five-LeVel Tree-Structured 32-Channel Linear-Phase Triplet-Based Wavelet Bases in EXample 4

\begin{tabular}{c|c|c|c|c|c}
\hline \hline & Level 1 & Level 2 & Level 3 & Level 4 & Level 5 \\
\hline Transition Bandwidth $\Delta \omega^{(l)}$ & $0.025 \pi$ & $0.05 \pi$ & $0.1 \pi$ & $0.2 \pi$ & $0.4 \pi$ \\
\hline Group Delay of $H_{0}^{(l)}(z), H_{1}^{(l)}(z)$ & 131,208 & 73,112 & 41,62 & 23,36 & 15,22 \\
\hline Passband deviation (Stopband & 0.2789 & 0.1728 & 0.1262 & 0.1382 & 0.00842 \\
attenuation) of $H_{0}^{(l)}(z) / \mathrm{dB}$ & $(37.43)$ & $(37.81)$ & $(39.51)$ & $(42.21)$ & $(39.46)$ \\
\hline Passband deviation (Stopband & 0.2773 & 0.1731 & 0.1255 & 0.1365 & 0.00921 \\
attenuation) of $H_{1}^{(l)}(z) / \mathrm{dB}$ & $(37.11)$ & $(37.34)$ & $(39.02)$ & $(43.96)$ & $(41.23)$ \\
\hline
\end{tabular}

TABLE VII

SOPOT COEFFICIENTS OF $R_{q i}^{(1)}(z), i=0,1,2$, FOR MULTIPLIERLESS FOUR-CHANNEL WAVELET FB IN EXAMPLE 5

\begin{tabular}{c|c|c|c}
\hline \hline $\boldsymbol{n}$ & $r_{0, n}^{(1)}$ & $r_{1, n}^{(1)}$ & $r_{2, n}^{(1)}$ \\
\hline 1 & $-2^{5}+2^{2}+2^{0}+2^{-3}+2^{-4}$ & $-2^{5}-2^{3}-2^{2}-2^{-1}-2^{-3}-2^{-4}$ & $-2^{4}+2^{0}+2^{-1}+2^{-4}$ \\
\hline 2 & $2^{6}+2^{5}-2^{1}-2^{0}-2^{-3}-2^{-4}$ & $2^{7}+2^{4}+2^{3}-2^{-4}$ & $2^{5}+2^{4}+2^{1}+2^{0}+2^{-3}+2^{-4}$ \\
\hline 3 & $-2^{7}+2^{4}+2^{1}+2^{-2}+2^{-4}$ & $-2^{7}-2^{5}-2^{4}+2^{-1}+2^{-3}+2^{-4}$ & $-2^{6}+2^{0}+2^{-1}-2^{-4}$ \\
\hline 4 & $2^{5}+2^{3}+2^{2}+2^{-1}+2^{-3}+2^{-4}$ & $2^{6}+2^{2}+2^{0}+2^{-4}$ & $2^{5}-2^{2}-2^{0}-2^{-3}-2^{-4}$ \\
\hline \hline
\end{tabular}

TABLE VIII

SOPOT COEFFICIENTS OF $Q_{q i}^{(1)}(z), i=0,1,2$, FOR MUlTIPLIERLESS Four-CHANNEL WAVELET FB IN EXAMPLE 5

\begin{tabular}{|c|c|c|c|}
\hline$n$ & $s_{0, n}^{(1)}$ & $s_{1, n}^{(1)}$ & $s_{2, n}^{(1)}$ \\
\hline 1 & $2^{5}-2^{2}-2^{0}-2^{-3}-2^{-4}-2^{-6}+2^{-11}+2^{-12}$ & $2^{5}+2^{3}+2^{2}+2^{-1}+2^{-3}+2^{-4}+2^{-7}+2^{-8}-2^{-12}$ & $2^{4}-2^{0}-2^{-1}-2^{-5}-2^{-6}-2^{-9}$ \\
\hline 2 & $2^{4}-2^{0}-2^{-1}-2^{-4}-2^{-5}-2^{-8}+2^{-11}$ & $2^{5}-2^{2}-2^{0}-2^{-3}-2^{-5}$ & $2^{2}+2^{1}+2^{-1}+2^{-4}+2^{-5}-2^{-12}$ \\
\hline 3 & $2^{2}+2^{1}+2^{-1}-2^{-7}-2^{-9}-2^{-11}$ & $2^{4}-2^{0}-2^{-1}+2^{-7}-2^{-10}$ & $2^{1}+2^{-2}+2^{-7}+2^{-10}$ \\
\hline 4 & $2^{1}+2^{-4}+2^{-6}+2^{-7}+2^{-10}+2^{-11}$ & $2^{3}-2^{0}-2^{-2}-2^{-4}-2^{-6}-2^{-9}-2^{-10}-2^{-12}$ & $2^{-2}+2^{-3}+2^{-5}+2^{-8}+2^{-9}+2^{-12}$ \\
\hline 5 & $2^{-3}+2^{-5}+2^{-8}+2^{-9}+2^{-11}$ & $2^{1}+2^{-2}+2^{-4}+2^{-5}+2^{-8}+2^{-10}+2^{-12}$ & $2^{-3}-2^{-6}-2^{-9}-2^{-11}$ \\
\hline 6 & $-2^{-2}+2^{-5}+2^{-9}+2^{-11}$ & $2^{-1}+2^{-8}+2^{-10}$ & $2^{-2}-2^{-5}+2^{-9}$ \\
\hline 7 & $-2^{-3}-2^{-5}-2^{-6}$ & $2^{-3}+2^{-4}+2^{-7}-2^{-10}-2^{-12}$ & $2^{-2}-2^{-6}-2^{-8}-2^{-13}$ \\
\hline 8 & $-2^{-2}+2^{-5}+2^{-7}+2^{-11}+2^{-12}$ & $2^{-2}+2^{-5}+2^{-7}$ & $2^{-2}+2^{-5}+2^{-8}+2^{-13}$ \\
\hline 9 & $-2^{-3}-2^{-4}-2^{-12}$ & $2^{-2}+2^{-6}+2^{-7}+2^{-9}+2^{-12}$ & $2^{-2}+2^{-5}+2^{-7}+2^{-9}+2^{-12}$ \\
\hline 10 & $-2^{-3}-2^{-4}+2^{-9}+2^{-12}$ & $2^{-2}+2^{-5}+2^{-6}+2^{-9}+2^{-11}$ & $2^{-2}+2^{-4}-2^{-9}$ \\
\hline 11 & $-2^{-3}-2^{-6}-2^{-7}-2^{-9}$ & $2^{-2}+2^{-6}+2^{-7}+2^{-9}+2^{-11}$ & $2^{-2}+2^{-5}+2^{-7}+2^{-9}$ \\
\hline 12 & $-2^{-3}-2^{-10}$ & $2^{-2}+2^{-6}-2^{-11}$ & $2^{-2}+2^{-6}+2^{-7}+2^{-10}+2^{-11}$ \\
\hline 13 & $-2^{-4}-2^{-6}-2^{-7}$ & $2^{-2}-2^{-6}-2^{-7}-2^{-10}-2^{-11}$ & $2^{-2}-2^{-6}-2^{-8}-2^{-10}$ \\
\hline 14 & $-2^{-4}+2^{-8}+2^{-10}$ & $2^{-3}+2^{-4}+2^{-8}+2^{-9}+2^{-12}$ & $2^{-3}+2^{-4}$ \\
\hline 15 & $-2^{-5}+2^{-8}+2^{-12}$ & $2^{-3}+2^{-6}+2^{-8}+2^{-9}$ & $2^{-3}+2^{-8}+2^{-9}+2^{-12}$ \\
\hline 16 & $-2^{-7}-2^{-8}+2^{-11}$ & $2^{-3}-2^{-6}+2^{-11}+2^{-12}$ & $2^{-4}+2^{-6}+2^{-7}-2^{-11}$ \\
\hline 17 & & $2^{-4}+2^{-7}-2^{-11}$ & $2^{-5}+2^{-7}+2^{-9}$ \\
\hline 18 & & $2^{-5}+2^{-7}+2^{-8}-2^{-12}$ & $2^{-6}-2^{-11}$ \\
\hline 19 & & $2^{-6}+2^{-8}-2^{-12}$ & \\
\hline 20 & & $2^{-7}-2^{-12}$ & \\
\hline
\end{tabular}

that the above relationship between $p_{i}$ 's can be maintained. Therefore, even though the zeros of $H_{m}(z)$ are properly imposed, the $K$ regularity condition can only be achieved by real-valued $p$. Another possibility is to allow $p_{i}$ 's to be rational numbers so that only simple integer divisions are involved. For simplicity, a longer wordlength for $p_{i}$ 's is chosen here to limit this error to an arbitrary low level. An example is now given to illustrate this effect.

Example 5. Multiplierless Four-Channel Triplet-Based Wavelet FBs: In this example, an four-channel multiplierless PR triplet-based FIR wavelet FB is designed. The specifications are identical to Example 3. With the use of (4.3), the remainder coefficients in Tables VII and IX, are obtained. To determine the SOPOT coefficients of the quotient coefficients using the random search algorithm, an objective function is performed to minimize the number of SOPOT terms while satisfying the given specifications. The target stopband attenuation of the analysis filter pair is $38 \mathrm{~dB}$. The optimized SOPOT coefficients of $Q_{q i}^{(l)}(z)$ are given in Tables VIII and $\mathrm{X}$ and the frequency response of the multiplierless FB is shown in Fig. 8(a)-(c). The passband deviation (stopband attenuation) of $H_{0}^{(1)}(z)$ and $H_{0}^{(2)}(z)$ are 0.0731 (38.66) dB and 0.0718 (39.01) dB, respectively. For $H_{1}^{(1)}(z)$ and $H_{1}^{(2)}(z)$, they are 0.0728 (38.03) $\mathrm{dB}$ and $0.0852(39.72) \mathrm{dB}$. It can be seen that the error at $\pi / 2$ is below $-120 \mathrm{~dB}$, when the wordlength of $p_{i}$ 's are chosen as 16 bits. The number of adders required to synthesize $Q_{q i}^{(l)}(z)$, without using MB, are 179 and 31, respectively. After using $\mathrm{MB}$, the number of adders is reduced to 92 and 20, respectively. The multiplierless four-channel FB has a stopband attenuation of approximately $38 \mathrm{~dB}$ and its phase response is approximately linear in the passband (not shown here). The corresponding analysis scaling function in Fig. 8(d) and the wavelet functions (not shown here) are also quite smooth even though $p$ is implemented as a 16-bit fixed-point number. 
TABLE IX

SOPOT COEFFICIENTS OF $R_{q i}^{(2)}(z), i=0,1,2$, FOR MULTIPLIERLESS FOUR-CHANNEL WAVELET FB IN EXAMPLE 5

\begin{tabular}{c|c|c|c}
\hline \hline $\boldsymbol{n}$ & $r_{0, n}^{(2)}$ & $r_{1, n}^{(2)}$ & $r_{2, n}^{(2)}$ \\
\hline 1 & $-2^{-2}-2^{-4}$ & $-2^{1}-2^{-3}-2^{-4}$ & $-2^{1}-2^{-3}-2^{-4}$ \\
\hline 2 & $2^{0}+2^{-2}+2^{-4}$ & $2^{3}+2^{-1}-2^{-4}$ & $2^{3}+2^{-1}-2^{-4}$ \\
\hline 3 & $-2^{1}-2^{-3}-2^{-4}$ & $-2^{3}-2^{2}+2^{-3}+2^{-4}$ & $-2^{3}-2^{2}+2^{-3}+2^{-4}$ \\
\hline 4 & $2^{1}+2^{-3}+2^{-4}$ & $2^{2}+2^{1}+2^{-1}+2^{-4}$ & $2^{2}+2^{1}+2^{-1}+2^{-4}$ \\
\hline
\end{tabular}

TABLE $\mathrm{X}$

SOPOT COEFFICIENTS of $Q_{q i}^{(2)}(z), i=0,1,2$, FOR MULTIPLIERLESS Four-CHANNEL WAVELET FB IN EXAMPLE 5

\begin{tabular}{c|c|c|c}
\hline $\boldsymbol{n}$ & $\boldsymbol{s}_{0, n}^{(2)}$ & $S_{1, n}^{(2)}$ & $s_{2, n}^{(2)}$ \\
\hline 1 & $2^{-2}+2^{-5}+2^{-7}$ & $2^{1}+2^{-3}+2^{-4}+2^{-7}+2^{-8}$ & $2^{1}+2^{-3}+2^{-4}+2^{-7}$ \\
\hline 2 & $-2^{-4}-2^{-7}-2^{-8}$ & $2^{-2}+2^{-4}+2^{-6}-2^{-9}$ & $2^{-2}+2^{-4}+2^{-6}$ \\
\hline 3 & $-2^{-6}$ & $-2^{-6}-2^{-10}$ & $-2^{-6}+2^{-9}$ \\
\hline 4 & $-2^{-5}-2^{-7}-2^{-8}$ & $2^{-4}-2^{-10}$ & $2^{-4}+2^{-7}$ \\
\hline 5 & $-2^{-6}-2^{-9}$ & $2^{-5}-2^{-9}$ & $2^{-5}+2^{-8}$ \\
\hline 6 & $-2^{-6}+2^{-10}$ & $2^{-5}+2^{-6}$ & $2^{-4}-2^{-8}-2^{-9}$ \\
\hline 7 & & $2^{-6}+2^{-7}$ & $2^{-5}-2^{-8}$ \\
\hline 8 & & $2^{-6}+2^{-7}+2^{-9}$ & $2^{-5}-2^{-8}$ \\
\hline 9 & & $2^{-7}+2^{-9}$ & $2^{-7}$ \\
\hline 10 & & $2^{-7}$ & $2^{-7}$ \\
\hline \hline
\end{tabular}

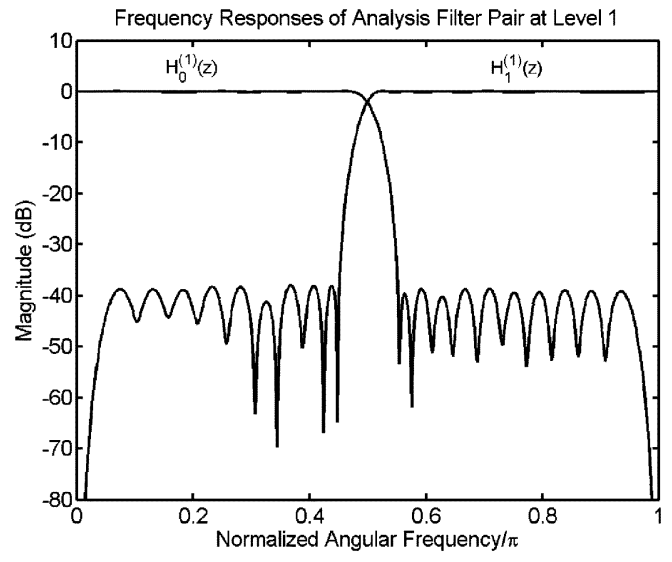

(a)

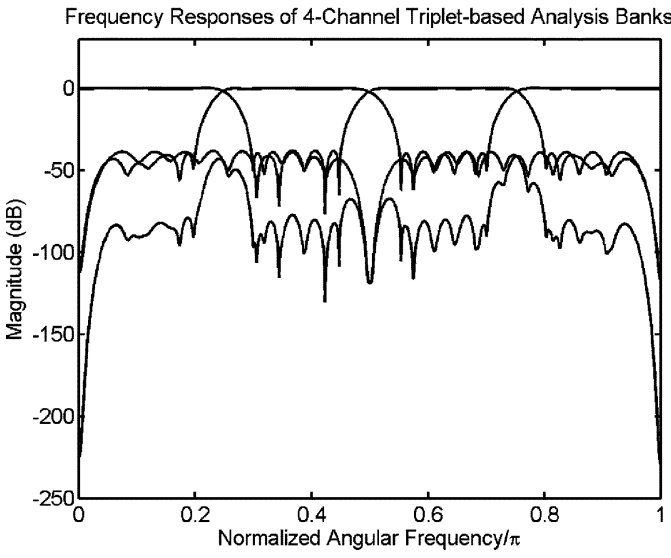

(c)

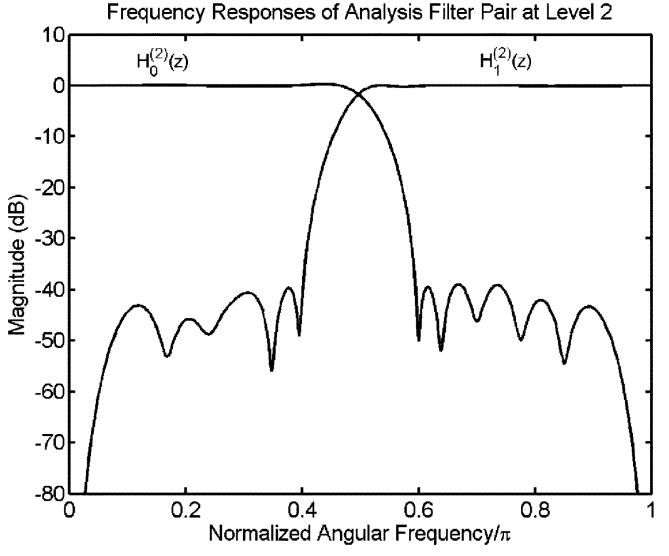

(b)

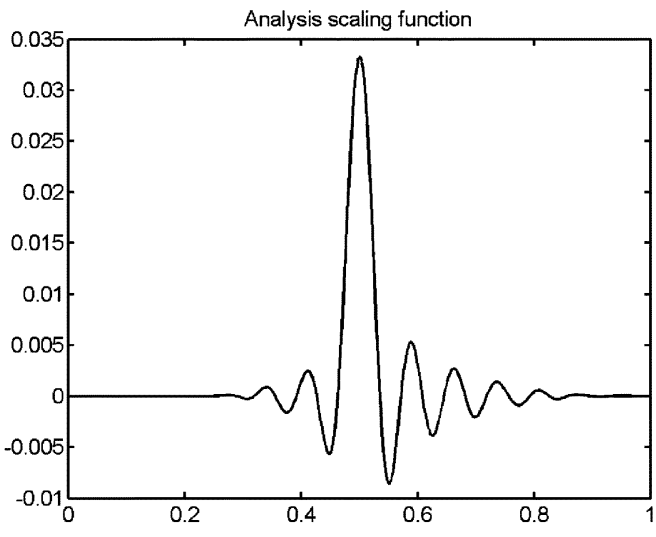

(d)

Fig. 8. Design results of the multiplierless four-channel triplet-based FB in Example 5: Frequency responses of the analysis filter pair at (a) level 1 and (b) level 2 of the tree. (c) Frequency responses of the multiplierless four-channel analysis banks. (d) Analysis scaling function. (Originally $\left.K_{0}=K_{1}=4\right)$.

\section{CONCLUSION}

New methods for the efficient design and realization of PR two-channel triplet-based FIR FBs and wavelet bases are pre- sented. The design problem is formulated as an SDP problem, while the $K$ regularity conditions are expressed as a set of linear equality constraints and they are structurally imposed into the design problem by eliminating the redundant variables. 
Design examples demonstrate the effectiveness of the proposed method for both linear-phase and low-delay FIR triplet FB. The more symmetric frequency response of the triplet FBs is exploited to construct a class of PR $M$-channel uniform FBs and wavelets with $M=2^{L}$ using a particular tree structure. The filter lengths of the two-channel FBs down the tree are approximately reduced by a factor of two at each level, while the transition bandwidths are successively increased by the same factor. This triplet-based uniform $M$-channel FB has very low design complexity and the PR condition and $K$ regularity conditions are structurally imposed. It has considerably lower arithmetic complexity and system delay than conventional tree structure using an identical FB. The multiplierless realization of these FBs using SOPOT coefficients and MB are also presented. Using a new factorization for structurally imposing the zeros of the subfilters, new multiplierless approximation of the triplet-based $M$-channel wavelet FBs with SOPOT coefficients are obtained.

\section{REFERENCES}

[1] S. M. Phoong, C. W. Lim, P. P. Vaidyanathan, and R. Ansari, "A new class of two-channel biorthogonal filter banks and wavelet bases," IEEE Trans. Signal Processing, vol. 43, pp. 649-664, Mar. 1995.

[2] J. S. Mao, S. C. Chan, W. Liu, and K. L. Ho, "Design and multiplierless implementation of a class of two-channel PR FIR filterbanks and wavelets with low system delay," IEEE Trans. Signal Processing, vol. 48, pp. 3379-3394, Dec. 2000.

[3] S. C. Chan, C. K. S. Pun, and K. L. Ho, "The design of a class of perfect reconstruction two-channel FIR and wavelets filterbanks using constrained least-squares method and semidefinite programming," in Proc. DSP'02, vol. 2, 2002, pp. 497-500.

[4] T. Cooklev and A. Nishihara, "Biorthogonal coiflets," IEEE Trans. Signal Processing, vol. 47, pp. 2582-2588, Sept. 1999.

[5] W. S. Lu and A. Antoniou, "Design of digital filters and filter banks by optimization: A state of the art review," in Proc. EUSIPCO'00, vol. 1, Sept. 2000, p. 78.

[6] S. C. Pei and P. H. Wang, "Closed-form design and efficient implementation of generalized maximally flat half-band FIR filters," IEEE Signal Processing Lett., vol. 7, pp. 149-151, Jun. 2000.

[7] W. Liu, S. C. Chan, and K. L. Ho, "Low-delay perfect reconstruction two-channel FIR/IIR filter banks and wavelet bases with SOPOT coefficients," in Proc. ICASSP'00, vol. 1, May 2000, pp. 109-112.

[8] D. B. H. Tay, "Least-squares design of the class of triplet halfband filter banks," in Proc. ISCAS'01, vol. 2, 2001, pp. 481-484.

[9] R. Ansari, C. W. Kim, and M. Dedovic, "Structure and design of twochannel filter banks derived from a triplet of halfband filters," IEEE Trans. Circuits Systs. II, vol. 46, pp. 1487-1496, Dec. 1999.

[10] Y. Nesterov and A. Nemirovskii, Interior Point Polynomial Methods in Convex Programming. Philadelphia, PA: SIAM, 1994.

[11] H. Wolkowicz, R. Saigal, and L. Vandenberghe, Handbook of Semidefinite Programming - Theory, Algorithms, and Application. Boston, MA: Kluwer, 2000.

[12] A. K. Soman, P. P. Vaidyanathan, and T. Q. Nquyen, "Linear-phase paraunitary filter banks: Theory, factorizations and designs," IEEE Trans. Signal Processing, vol. 41, pp. 3480-3496, Dec. 1993.

[13] S. C. Chan, Y. Luo, and K. L. Ho, "M-channel compactly supported biorthogonal cosine-modulated wavelet bases," IEEE Trans. Signal Processing, vol. 46, pp. 1142-1151, 1998.

[14] P. P. Vaidyanathan, Multirate Systems and Filter Banks. Englewood Cliffs, NJ: Prentice Hall, 1993.

[15] X. M. Xie, S. C. Chan, and T. I. Yuk, "M-band perfect-reconstruction linear-phase filter banks," in Proc. 11th IEEE Signal Processing Workshop on Statistical Signal Processing, 2001, pp. 583-586.

[16] M. J. T. Smith and T. P. Barnwell III, "Exact reconstruction techniques for tree-structured subband coders," IEEE Trans. Acoust., Speech, Signal Processing, vol. ASSP-34, pp. 434-441, June 1986.
[17] N. J. Fliege, Multirate Digital Signal Processing. New York: Wiley, 1995.

[18] K. Nayebi, T. P. Barnwell III, and M. J. T. Smith, "Time-domain filter bank analysis: A new design theory," IEEE Trans. Signal Processing, vol. 40, pp. 1412-1429, June 1992.

[19] T. Q. Nguyen and P. P. Vaidyanathan, "Two-channel perfect-reconstruction FIR QMF structures which yield linear-phase analysis and synthesis filters," IEEE Trans. Signal Processing, vol. 37, pp. 676-690, May 1989.

[20] C. Herley and M. Vetterli, "Orthogonal time-varying filter banks and wavelet packets," IEEE Trans. Signal Processing, vol. 42, pp. 2650-2663, Oct. 1994.

[21] K. S. Yeung and S. C. Chan, "The design of two-channel perfect reconstruction FIR triplet wavelet filter banks using semidefinite programming," in Proc. ISCAS'03, vol. 4, May 2003, pp. 253-256.

[22] W. Sweldens, "The lifting scheme: A new philosophy in biorthogonal wavelet construction," in Proc. SPIE Wavelet Applications in Signal and Image Processing III, vol. 2569, July 1995, pp. 68-79.

[23] Y. C. Lim and S. R. Parker, "FIR filter design over a discrete power-of-two coefficient space," IEEE Trans. Acoust., Speech, Signal Processing, vol. ASSP-31, pp. 583-591, Apr. 1983.

[24] G. Wade, A. Roberts, and G. Williams, "Multiplierless FIR filter design using a genetic algorithm," IEEE Vision, Image, Signal Processing, vol. 141, pp. 175-180, June 1994.

[25] C. K. S. Pun, S. C. Chan, and K. L. Ho, "Efficient design of a class of multiplierless perfect reconstruction two-channel filter banks and wavelets with prescribed output accuracy," in Proc. 11th IEEE Signal Processing Workshop on Statistical Signal Processing, 2001, pp. 599-602.

[26] C. C. Chen and A. N. Willson, "A trellis search algorithm for the design of FIR filters with signed-powers-of-two coefficients," IEEE Trans. Circuits Syst. II, vol. 46, pp. 29-39, Jan. 1999.

[27] A. G. Dempster and M. D. MacLeod, "Use of minimum-adder multiplier blocks in FIR digital filters," IEEE Trans. Circuits Syst. II, vol. 42, pp. 569-577, Sept. 1995.

[28] I. Daubechies, "Othonormal bases of compactly supported wavelets," Commun. Pure Appl. Math., vol. 41, pp. 909-996, 1988.

[29] S. C. Chan, C. K. S. Pun, and K. L. Ho, "The design of a class of perfect reconstruction two-channel FIR linear-phase filterbanks and wavelets bases using semidefinite programming," IEEE Signal Processing Lett., vol. 11, pp. 297-300, Feb. 2004, to be published.

[30] C. K. S. Pun, "New Design and realization techniques for prefect reconstruction two-channel filterbanks and wavelets bases," M.Phil. thesis, The University of Hong Kong, Hong Kong, 2002.

[31] W. S. Lu, "Design of nonlinear-phase FIR digital filters: A semidefinite programming approach," in Proc. ISCAS'99, vol. 3, May 1999, pp. 263-266.

[32] S. P. Wu, S. Boyd, and L. Vandenberghe, "FIR filter design via semidefinite programming and spectral factorization," in Proc. 5th Conf. Decision and Control, Dec. 1996, pp. 271-276.

[33] W. S. Lu, "Design of 2-D FIR filters with power-of-two coefficients: A semidefinite programming relaxation approach," in Proc. ISCAS'01, vol. 2, May 2001, pp. 549-552.

[34] - "Design of stable minimax IIR digital filters using semidefinite programming," in Proc. ISCAS'00, vol. 1, May 2000, pp. 355-358.

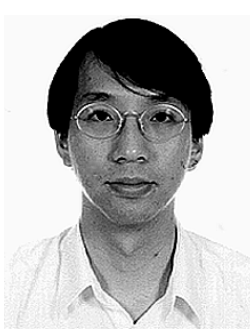

S. C. Chan (S'87-M'92) received the B.Sc. (Eng.) and $\mathrm{Ph}$.D. degrees from the University of Hong Kong, Hong Kong, in 1986 and 1992, respectively.

In 1990, he joined the City Polytechnic of Hong Kong, Hong Kong, as an Assistant Lecturer where he later was a University Lecturer. Since 1994, he has been with the Department of Electrical and Electronic Engineering, the University of Hong Kong, where he is now an Associate Professor. He was a Visiting Researcher at Microsoft Corporation, Redmond, CA and Microsoft Asia, Beijing, China in 1998 and 1999, respectively. His research interests include fast transform algorithms, filter design and realization, multirate signal processing, communications signal processing, and image-based rendering.

Dr. Chan is currently a member of the Digital Signal Processing Technical Committee of the IEEE Circuits and Systems Society. He was Chairman of the IEEE Hong Kong Chapter of Signal Processing from 2000 to 2002. 


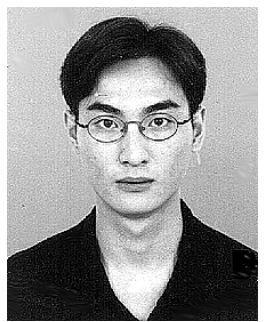

K. S. Yeung received the B.Eng. degree in electrical and electronic engineering from The University of Hong Kong, Hong Kong, in 2001. He is currently pursuing the M.Phil. degree in electrical and electronic engineering at the same university.

His main research interests are in digital signal processing, multirate filter banks and wavelets, digital filter design, and their efficient realization and applications. 